\title{
Nitrate supplementation improves physical performance specifically in non-athletes during prolonged open-ended tests: a systematic review and meta-analysis
}

\author{
Helton O. Campos $^{1} \dagger$, Lucas R. Drummond ${ }^{1} \dagger$, Quezia T. Rodrigues ${ }^{1}$, Frederico S. M. Machado ${ }^{1}$, \\ Washington Pires ${ }^{2}$, Samuel P. Wanner ${ }^{3}$ and Cândido C. Coimbra ${ }^{1 *}$ \\ ${ }^{1}$ Departamento de Fisiologia e Biofísica, Instituto de Ciências Biológicas, Universidade Federal de Minas Gerais, Av. Antônio \\ Carlos 6627, 31270-901, Belo Horizonte, Minas Gerais, Brazil \\ ${ }^{2}$ Departamento de Educação Física, Universidade Federal de Juiz de Fora - Campus Governador Valadares, Av. Doutor \\ Raimundo Monteiro Rezende 330, 35010-177, Governador Valadares, Minas Gerais, Brazil \\ ${ }^{3}$ Departamento de Educação Física, Universidade Federal de Minas Gerais, Av. Antônio Carlos 6627, 31270-901, \\ Belo Horizonte, Minas Gerais, Brazil
}

(Submitted 31 March 2017 - Final revision received 6 December 2017 - Accepted 5 January 2018)

\section{Abstract}

Nitrate $\left(\mathrm{NO}_{3}^{-}\right)$is an ergogenic nutritional supplement that is widely used to improve physical performance. However, the effectiveness of $\mathrm{NO}_{3}^{-}$ supplementation has not been systematically investigated in individuals with different physical fitness levels. The present study analysed whether different fitness levels (non-athletes $v$. athletes or classification of performance levels), duration of the test used to measure performance (short $v$. long duration) and the test protocol (time trials $v$. open-ended tests $v$. graded-exercise tests) influence the effects of $\mathrm{NO}_{3}^{-}$ supplementation on performance. This systematic review and meta-analysis was conducted and reported according to the guidelines outlined in the Preferred Reporting Items for Systematic Reviews and Meta-Analysis (PRISMA) statement. A systematic search of electronic databases, including PubMed, Web of Science, SPORTDiscus and ProQuest, was performed in August 2017. On the basis of the search and inclusion criteria, fifty-four and fifty-three placebo-controlled studies evaluating the effects of $\mathrm{NO}_{3}^{-}$supplementation on performance in humans were included in the systematic review and meta-analysis, respectively. $\mathrm{NO}_{3}^{-}$supplementation was ergogenic in non-athletes (mean effect size (ES) $0 \cdot 25 ; 95 \%$ CI $0 \cdot 11,0 \cdot 38$ ), particularly in evaluations of performance using long-duration open-ended tests (ES $0.47 ; 95 \%$ CI $0 \cdot 23$, $0 \cdot 71$ ). In contrast, $\mathrm{NO}_{3}^{-}$supplementation did not enhance the performance of athletes (ES $0 \cdot 04 ; 95 \% \mathrm{CI}-0 \cdot 05,0 \cdot 15$ ). After objectively classifying the participants into different performance levels, the frequency of trials showing ergogenic effects in individuals classified at lower levels was higher than that in individuals classified at higher levels. Thus, the present study indicates that dietary $\mathrm{NO}_{3}^{-}$supplementation improves physical performance in non-athletes, particularly during long-duration open-ended tests.

Key words: Diet: Fitness level: Nitric oxide: Fatigue

Nitrate $\left(\mathrm{NO}_{3}^{-}\right)$is an ergogenic nutritional supplement widely consumed by exercise practitioners and athletes to improve their health and physical performance ${ }^{(1)}$. The widespread use of $\mathrm{NO}_{3}^{-}$likely reflects its abundant availability in many vegetables, and its content ranges from $<20 \mathrm{mg} / 100 \mathrm{~g}$ in sweet potato to $>250 \mathrm{mg} / 100 \mathrm{~g}$ in beetroot ${ }^{(2)}$. Although oral bacteria can reduce $\mathrm{NO}_{3}^{-}$to nitrite $\left(\mathrm{NO}_{2}^{-}\right)$, the transit of these foods in the mouth is short, and the resulting increase in $\mathrm{NO}_{3}^{-}$bioavailability appears to be related to the intrinsic $\mathrm{NO}_{3}^{-}$content in the vegetable or supplement. Indeed, increased $\mathrm{NO}_{3}^{-}$bioavailability could favour nitric oxide (NO) synthesis ${ }^{(3)}$. NO is a signalling molecule associated with improved cardiovascular and skeletal muscle functions that may potentially enhance physical performance and even facilitate adaptations to exercise training ${ }^{(4)}$. Nevertheless, the scientific literature provides controversial results regarding the performance-enhancing effects induced by $\mathrm{NO}_{3}^{-}$supplementation.

Two systematic reviews and meta-analyses on this topic have recently been published, establishing clear practical recommendations and directions for future studies investigating changes in performance induced by $\mathrm{NO}_{3}^{-}$supplementation ${ }^{(5,6)}$. Hoon et $a l^{(5)}$ and McMahon et al. ${ }^{(6)}$ analysed data according to the exercise protocol used (i.e. time trials, open-ended tests and graded-exercise tests) and observed that dietary $\mathrm{NO}_{3}^{-}$supplementation improved endurance only when performance was evaluated using open-ended tests. Notably, none of these two

Abbreviation: PL, performance level.

* Corresponding author: C. C. Coimbra, fax +55 313409 2924, email coimbrac@icb.ufmg.br

$\dagger$ These authors contributed equally to this work. 
meta-analyses divided and analysed separately the studies conducted with athletes or non-athletes, as we are proposing here. McMahon et al. performed a continuous variable metaregression analysis and reported that the fitness level did not have an influence on the ergogenic effect of dietary $\mathrm{NO}_{3}^{-}$ supplementation ${ }^{(6)}$. However, grouping the data according to the exercise protocol may result in an important bias. In fact, the studies using open-ended tests were mainly performed in non-athletes. In contrast, most studies using time trials were performed in athletes. This disparity might have led to a misinterpretation of the results owing to an unintentional division based on individuals' physical fitness level. Interestingly, neither of the two recent systematic reviews addressed the following question raised by Jonvik et al: 'Can elite athletes benefit from dietary nitrate supplementation? ${ }^{(7-9)}$. Therefore, information regarding the effectiveness of $\mathrm{NO}_{3}^{-}$supplementation in individuals with different physical fitness levels is lacking. Moreover, physical performance is modulated by various mechanisms and depends on several factors, including the duration of the test performed (i.e. short or long duration). Thus, the influence of the test duration on the changes in performance induced by $\mathrm{NO}_{3}^{-}$supplementation in individuals with different fitness levels remains to be investigated.

Increased $\mathrm{NO}$ availability resulting from $\mathrm{NO}_{3}^{-}$supplementation has beneficial effects on health and physical performance and has been largely studied in humans and laboratory animals. In the central nervous system, NO prevented exaggerated increases in the core body temperature in rats subjected to exercise by increasing cutaneous heat loss and decreasing the metabolic cost of running ${ }^{(10-13)}$. In these rat studies, the pharmacological blockade of central NO synthesis markedly impaired endurance $^{(10,12)}$, whereas an increased NO availability in the brain did not affect endurance ${ }^{(13)}$. In humans, the physical performance benefits mediated by dietary $\mathrm{NO}_{3}^{-}$supplementation have been attributed to peripheral effects, including reduced arterial pressure and $\mathrm{VO}_{2}$. The latter effect leads to a reduced oxygen cost during exercise that is most likely due to the reduced cost of ATP for muscle force production, improved mitochondrial efficiency and increased muscle oxygenation ${ }^{(14,15)}$. In contrast, the adverse events related to $\mathrm{NO}_{3}^{-}$supplementation are minor and restricted to red urine (beeturia) and stool, which usually results from the ingestion of beetroot in juice or meals ${ }^{(16,17)}$

Interestingly, both acute and chronic supplementations of $\mathrm{NO}_{3}^{-}$have been shown to either improve ${ }^{(18-24)}$ or have no effect $^{(14,25-29)}$ on endurance performance. The uncertain efficacy of $\mathrm{NO}_{3}^{-}$supplementation appears to be related to the fitness level of the investigated population as demonstrated by a careful evaluation of the cumulative number of trials reporting the performance benefits or lack thereof in both non-athletes (healthy individuals engaged in regular physical activity but not involved in sports competitions) and athletes (Fig. 1). Notably, nearly $65 \%$ of the publications on this topic did not report the benefits resulting from $\mathrm{NO}_{3}^{-}$supplementation. However, if only those studies performed in non-athletes are considered, approximately $45 \%$ of the publications show a supplementation-mediated positive effect on physical performance, whereas the percentage of papers showing beneficial effects in athletes is lower than $30 \%$ (Fig. 1). Collectively, these observations reinforce the

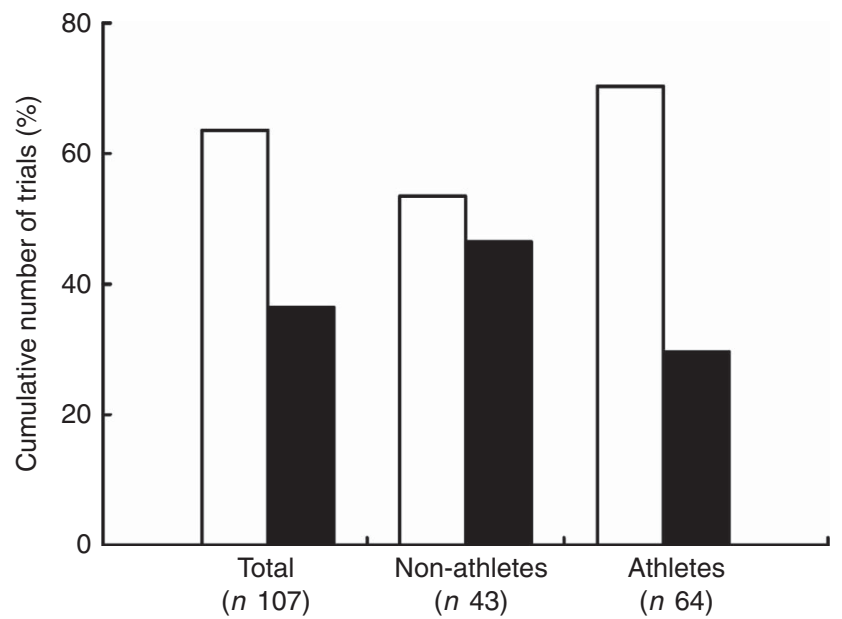

Fig. 1. Number of trials (\%) reporting that dietary $\mathrm{NO}_{3}^{-}$supplementation had no effect $(\square)$ and/or a positive effect $(\square)$ on physical performance in non-athletes and athletes.

relevance of the present systematic review and meta-analysis, which help clarify the contradictory reports of the effects of $\mathrm{NO}_{3}^{-}$supplementation on physical performance.

Therefore, the present study systematically analysed whether different physical fitness levels (i.e. non-athletes $v$. athletes) influence the effects of $\mathrm{NO}_{3}^{-}$supplementation on physical performance. In addition, we also evaluated the influence of the duration of the tests used to measure performance (i.e. short $v$. long duration) and the test protocol used (i.e. time trials $v$. openended tests $v$. graded-exercise tests) on the effect of $\mathrm{NO}_{3}^{-}$supplementation on physical performance in individuals with different physical fitness levels. Thus, the present analyses provide information that is useful to exercise practitioners, athletes, coaches and conditioning professionals who are interested in improving physical performance and achieving health benefits.

\section{Methods}

\section{Search strategy}

This systematic review and meta-analysis was conducted and reported according to the guidelines outlined in the Preferred Reporting Items for Systematic Reviews and Meta-Analysis (PRISMA) statement ${ }^{(30,31)}$. A systematic search of electronic databases, including PubMed, Web of Science, SPORTDiscus and ProQuest, was performed in August 2017 without any date restrictions. The search strategy was supplemented by manual cross-matching of each publication reference list and key author searches. Combinations of the following keywords were used: effort, endurance, exercise, fatigue, nitrate, nitrate supplementation, nitrite, nitrite supplementation, performance, power, running, speed, sport and workload.

\section{Study selection}

Studies that met the following criteria were included in this systematic review and meta-analysis: (i) the participants were healthy humans (either non-athletes or athletes), (ii) physical 
performance was measured after the participants were supplemented with $\mathrm{NO}_{3}^{-}$and (iii) the studies were placebocontrolled trials. Furthermore, all included studies were written in English. Reviews, summaries, case studies and letters were not included, although this bibliography was consulted. Studies involving hypoxic conditions, individuals with diseases, exercise in the heat, children and elderly people, and laboratory animals were excluded. On the basis of the search and inclusion/exclusion criteria, fifty-four studies (106 trials) were selected for inclusion in this systematic review, and fifty-three studies (104 trials) were included in the meta-analysis (Fig. 2). Notably, several studies measured more than one physical performance parameter. The data addressing the effect of $\mathrm{NO}_{3}^{-}$supplementation on each parameter were included, and therefore the number of trials was greater than the number of studies. Only one study with one trial $^{(20)}$ and one trial in a study with several trials ${ }^{(32)}$ were excluded from the meta-analysis because they did not include the standard deviation data needed to calculate the effect size.

\section{Data grouping}

The selected studies were divided into the following two groups according to the physical fitness level of the individuals tested: non-athletes (forty-three trials) and athletes (sixty-three trials). The individuals were allocated into these two groups according to the classification used by the authors of the research papers, which were consulted. This strategy was efficient in dividing the participants into two groups with different functional capacities as demonstrated by the higher $\mathrm{VO}_{2 \max }$ values in the athletes than in the non-athletes (61.1 (sD 1.8) $v .50 \cdot 5$ (SD 1.8) $\mathrm{ml} / \mathrm{kg}$ per min; $t$-test, $P<0.05$ ). Similarly, the studies selected for inclusion in the meta-analysis were initially divided into the following two groups: non-athletes (forty-three trials) and athletes (sixty-one trials). The two groups were then subdivided according to the duration of the test performed as follows: short duration (non-athletes, eighteen trials; athletes, seventeen trials) or long duration (non-athletes, twenty-five trials; athletes, forty-four trials). Exercises lasting less than $180 \mathrm{~s}$, thereby characterised by a relevant anaerobic contribution to the energy expenditure, were considered short-duration exercises. Alternatively, exercise bouts lasting more than $180 \mathrm{~s}$ were considered longduration exercises ${ }^{(33)}$. In addition, because $\mathrm{NO}_{3}^{-}$supplementation has been shown to have a positive effect on physical performance only in non-athletes during long-duration tests, this group was further subdivided according to the test protocol used (openended tests (constant power), fourteen trials; time trials, four trials; and graded-exercise tests (incremental power), five trials). Open-ended tests consist of exercising at a constant power until the participant is volitionally fatigued; the time until fatigue, which may be highly variable among subjects, is considered the main measure of performance in this test. Finally, owing to the large number of studies in cycling athletes, a specific analysis was conducted for this sport (thirty-seven trials).

Analysis of the relationship between the performance level and the response to $\mathrm{NO}_{3}^{-}$supplementation

Because the authors of the research papers may have been imprecise in the classification of their subjects as athletes, we decided to perform an objective analysis. Thus, the individuals were grouped into different performance levels (PL) according to the classification provided by De Pauw et al. ${ }^{(34)}$. These authors divided the participants in sport science studies into the following five different levels: performance level 1 (PL1) included untrained and sedentary subjects with a $\mathrm{VO}_{2 \max }<45.0 \mathrm{ml} / \mathrm{kg}$ per min; performance level 2 (PL2) included recreationally trained subjects with a $\mathrm{VO}_{2 \max }$ between 45.0 and $54.9 \mathrm{ml} / \mathrm{kg}$ per min; performance level 3 (PL3) included trained subjects with a $\mathrm{VO}_{2 \max }$ between 55.0 and $64.9 \mathrm{ml} / \mathrm{kg}$ per min; performance level 4 (PL4) included highly trained subjects with a $\mathrm{VO}_{2 \max }$ between 65.0 and $71.0 \mathrm{ml} / \mathrm{kg}$ per min; and performance level 5 (PL5) included professional subjects with a $\mathrm{VO}_{2 \max }>71.0 \mathrm{ml} / \mathrm{kg}$ per min. On the basis of this study, we grouped the individuals into five levels and then evaluated the relationship between the PL and the changes in performance induced by $\mathrm{NO}_{3}^{-}$supplementation.

\section{Risk of bias assessment}

Two independent reviewers assessed the risk of bias using an adapted Grading of Recommendations Assessment, Development and Evaluation (GRADE) instrument ${ }^{(35)}$. Discrepant evaluations were settled via discussion with a third reviewer. Using this approach, it was possible to evaluate the risk of bias in each study included in the present systematic review. Domains reflecting sequence generation, allocation concealment, blinding of participants and personnel, incomplete outcome data, selective outcome reporting and other sources of bias were evaluated.

\section{Statistical analysis}

The mean and standard deviation values of the performance indexes in both the $\mathrm{NO}_{3}^{-}$supplementation and control trials were obtained from the data provided in the consulted research papers. Heterogeneity was evaluated using the $\chi^{2}$ test for homogeneity and the $I^{2}$ statistic. The effect size (Cohen's $d$ or Hedges' $g$ ) was calculated for the performance indexes in each study. Then, a weighted-mean estimate of the effect size was calculated to account for differences in the sample sizes. The mean unweighted effect size and associated $95 \%$ CI were also calculated. We used Cohen's classification of the effect size magnitude, where $d<0.20$ for negligible effect; $d=0.20-0.49$ for small effect; $d=0.50-0.79$ for moderate effect; and $d>0.8$ for large effect ${ }^{(36)}$. The $\chi^{2}$ test was used to compare the frequency of trials showing improved performance in response to $\mathrm{NO}_{3}^{-}$supplementation among the different PL. Student's $t$ test was used to compare the $\mathrm{VO}_{2 \max }$ between the non-athletes and athletes. Pearson's correlations were performed to evaluate the association between the supplementation parameters (dose, number of days and total amount ingested) and the changes in physical performance. Publication bias was assessed by a visual inspection of funnel plots of the standard error $v$. effect size ${ }^{(37)}$.

\section{Results}

\section{Systematic review}

In total, 4732 studies were identified through the database and reference searches. After removing the duplicates and 


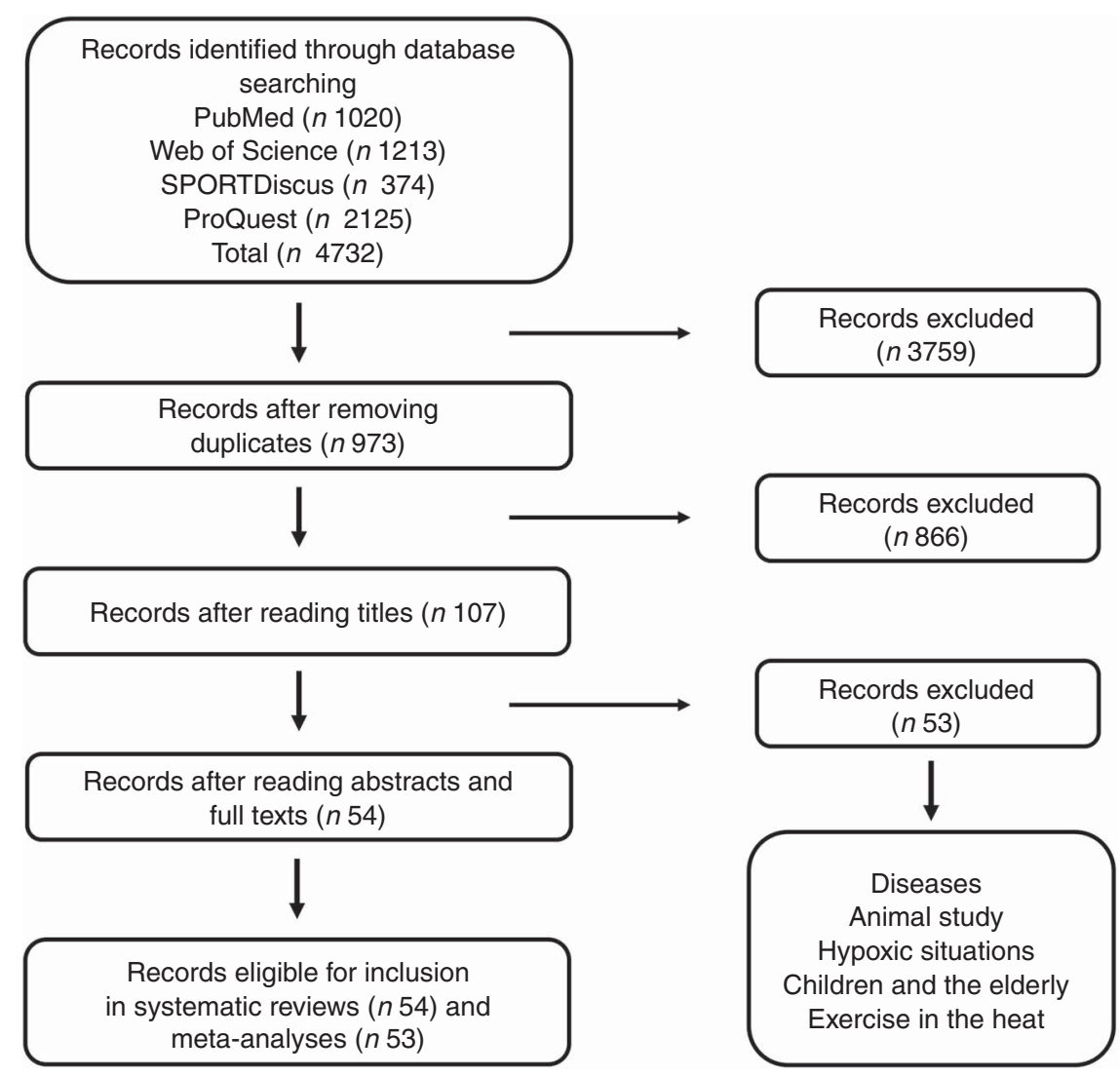

Fig. 2. Summary of the study selection process.

excluding papers that did not meet the eligibility criteria according to a review of their titles, abstracts and full texts, fiftyfour studies (106 trials and 662 individuals) were selected for inclusion in the systematic review (Fig. 2).

The characteristics of the subjects, including information regarding the supplementation regimens and effects of $\mathrm{NO}_{3}^{-}$ supplementation on the physical performance of non-athletes and athletes in each study, are summarised in Tables 1 and 2, respectively. Notably, most studies used beetroot juice as a form of $\mathrm{NO}_{3}^{-}$supplementation. However, these studies were heterogeneous in several supplementation features, including the ingested volume $(70,140,250,280$ or $500 \mathrm{ml}$ ), dose (between 4.0 and $19.5 \mathrm{mmol}$ ), days of supplementation (between 1 and $15 \mathrm{~d}$ ), timing of supplementation before the trial (between 40 and $1440 \mathrm{~min}$ ) and the parameter measured to determine physical performance.

\section{Meta-analyses}

In total, fifty-three studies (104 trials and 648 individuals) were included in the meta-analysis.

Non-athletes. After pooling the data from forty-three trials, the mean effect size was 0.25 (95\% CI 0.11, 0.38), which indicates that the dietary $\mathrm{NO}_{3}^{-}$supplementation had a small and significant beneficial effect on physical performance $(P<0.05$; Fig. 3). According to a fixed-effects analysis, no heterogeneity was observed among these studies $\left(I^{2}=0 \% ; Q=15 \cdot 26\right.$, $\mathrm{df}=42$, $P=1 \cdot 00)$.

Athletes. After pooling the data from sixty-one trials, the mean effect size was 0.04 (95\% CI $-0.05,0.15)$, which indicates that the dietary $\mathrm{NO}_{3}^{-}$supplementation had a negligible and nonsignificant effect on improving physical performance $(P>0.05$; Fig. 4). According to a fixed-effects analysis, no heterogeneity was observed among these studies $\left(I^{2}=0 \% ; Q=18 \cdot 16, \mathrm{df}=60\right.$, $P=1 \cdot 00)$. The subsequent analysis consisted of subdividing both the athletes and non-athletes into those performing shortand long-duration tests.

Non-athletes subjected to short-duration tests. After pooling the data from eighteen trials, the mean effect size was $0 \cdot 12$ (95\% CI $-0.07,0.31$ ), which indicates that the dietary $\mathrm{NO}_{3}^{-}$ supplementation had a negligible and non-significant effect on physical performance $(P>0.05$; Fig. 5$)$. According to a fixed-effects analysis, no heterogeneity was observed among these studies $\left(I^{2}=0 \% ; Q=4.43, \mathrm{df}=17, P=0.99\right)$.

Athletes subjected to short-duration tests. After pooling the data from seventeen trials, the mean effect size was 0.03 $(95 \%$ CI $-0 \cdot 17,0 \cdot 23$ ), which indicates that the dietary $\mathrm{NO}_{3}^{-}$supplementation had a negligible and non-significant effect on performance $(P>0.05$; Fig. 6). According to a random-effects 


\section{N British Journal of Nutrition}

Table 1. Study characteristics - non-athletes

(Mean values and standard deviations)

\begin{tabular}{|c|c|c|c|c|c|c|c|c|c|c|c|c|}
\hline \multirow[b]{3}{*}{ References } & \multirow{3}{*}{$\begin{array}{l}\text { No. of } \\
\text { subjects } \\
(0,+)\end{array}$} & \multirow{3}{*}{$\begin{array}{l}\text { Characteristics of } \\
\text { subjects }\end{array}$} & \multicolumn{7}{|c|}{ Nitrate supplementation } & \multirow{3}{*}{ Exercise protocol } & \multirow{3}{*}{$\begin{array}{l}\text { Variable of } \\
\text { physical } \\
\text { performance }\end{array}$} & \multirow[b]{3}{*}{ Results } \\
\hline & & & \multicolumn{2}{|c|}{$\begin{array}{l}\mathrm{VO}_{2 \text { peak }} / \mathrm{NO}_{2 \max } \\
(\mathrm{ml} / \mathrm{kg} \mathrm{per} \mathrm{min})\end{array}$} & \multirow{2}{*}{$\begin{array}{l}\text { Ingested fluid/ } \\
\text { volume (ml) }\end{array}$} & \multirow{2}{*}{$\begin{array}{l}\text { Dose } \\
(\mathrm{mmol})\end{array}$} & \multirow[b]{2}{*}{ Placebo substance } & \multirow{2}{*}{$\begin{array}{l}\text { Days of } \\
\text { supplementation }\end{array}$} & \multirow{2}{*}{$\begin{array}{l}\text { Time before } \\
\text { trial (min) }\end{array}$} & & & \\
\hline & & & Mean & SD & & & & & & & & \\
\hline $\begin{array}{l}\text { Aucouturier } \\
\text { et al. (1) }\end{array}$ & $12\left(0^{\star}\right)$ & Healthy & $46 \cdot 6$ & 3.4 & $\mathrm{BJ} / 500$ & 5.4 & $\begin{array}{l}\text { Apple blackcurrant } \\
\text { juice }\end{array}$ & 3 & 90 & $\begin{array}{l}\text { Supramaximal } \\
\text { intermittent exercise } \\
\text { test }\end{array}$ & Work (kJ) & $\begin{array}{l}S=168 \cdot 1(\text { SD } 60.2) \\
N S=142 \cdot 0(\text { SD } 46.8) \\
D=n o\end{array}$ \\
\hline $\begin{array}{l}\text { Aucouturier } \\
\text { et al. (2) }\end{array}$ & $12\left(0^{\star}\right)$ & Healthy & $46 \cdot 6$ & 3.4 & $\mathrm{BJ} / 500$ & 5.4 & $\begin{array}{l}\text { Apple blackcurrant } \\
\text { juice }\end{array}$ & 3 & 90 & $\begin{array}{l}\text { Supramaximal } \\
\text { intermittent exercise } \\
\text { test }\end{array}$ & Time $(\mathrm{min})$ & $\begin{array}{l}S=19 \cdot 6(\text { so } 8 \cdot 1) \\
N S=16 \cdot 4(\text { sD } 6 \cdot 0) \\
D=\text { yes }\end{array}$ \\
\hline $\begin{array}{l}\text { Bailey } \\
\text { et al. }{ }^{(18)}\end{array}$ & $8\left({ }^{(}\right)$ & Healthy & 49 & 5 & $\mathrm{BJ} / 500$ & 5.5 & $\begin{array}{l}\text { Blackcurrant cordial } \\
\text { without nitrate }\end{array}$ & 6 & NR & $\begin{array}{l}\text { Severe-intensity } \\
\text { exercise }\end{array}$ & Time (s) & $\begin{array}{l}S=675(\mathrm{SD} 203) \\
N S=583(\mathrm{SD} 145) \\
D=\text { yes }\end{array}$ \\
\hline $\begin{array}{l}\text { Bailey } \\
\text { et al. }{ }^{(19)}\end{array}$ & $7\left(0^{(0)}\right.$ & $\begin{array}{l}\text { Healthy, recreationally } \\
\text { active }\end{array}$ & & - & $\mathrm{BJ} / 500$ & $5 \cdot 1$ & $\begin{array}{l}\text { Low-energy } \\
\text { blackcurrant juice } \\
\text { cordial }\end{array}$ & 6 & NR & High-intensity exercise & Time (s) & $\begin{array}{l}S=734(\mathrm{sD} 288) \\
\mathrm{NS}=586(\mathrm{SD} 211) \\
\mathrm{D}=\mathrm{no}\end{array}$ \\
\hline $\begin{array}{l}\text { Bailey } \\
\quad \text { et al. (1) }\end{array}$ & $7($ () & Healthy & & - & $\mathrm{BJ} / 70$ & $6 \cdot 2$ & Sodium chloride & 9 & 150 & Cycling at $35 \mathrm{rpm}$ & Time (s) & $\begin{array}{l}S=344(\text { sD } 74) \\
N S=341(\text { SD } 99) \\
D=\text { no }\end{array}$ \\
\hline $\begin{array}{l}\text { Bailey } \\
\quad \text { et al. (2) }\end{array}$ & 7()$\left.^{*}\right)$ & Healthy & & - & $\mathrm{BJ} / 70$ & $6 \cdot 2$ & Sodium chloride & 9 & 150 & Cycling at $115 \mathrm{rpm}$ & Time (s) & $\begin{array}{l}S=362(\text { SD } 137) \\
N S=297(\text { SD } 79) \\
D=\text { yes }\end{array}$ \\
\hline $\begin{array}{l}\text { Breese } \\
\text { et al. }^{(40)}\end{array}$ & $\begin{array}{l}9\left(40^{+}\right. \\
\text {and } \\
50)\end{array}$ & $\begin{array}{l}\text { Healthy, physically } \\
\text { active }\end{array}$ & $\begin{array}{l}\delta=3.73 \\
q=2.69\end{array}$ & $\begin{array}{l}\delta=0.46^{\star} \\
\phi=0.52^{\star}\end{array}$ & $\mathrm{BJ} / 140$ & 8.0 & $\begin{array}{l}\text { BJ negligible nitrate } \\
\text { content }\end{array}$ & 6 & 120 & $\begin{array}{l}\text { Step exercise tests until } \\
\text { fatigue }\end{array}$ & Time (s) & $\begin{array}{l}S=635(\text { SD 258) } \\
N S=521(\text { SD 158) } \\
D=\text { yes }\end{array}$ \\
\hline Buck et al. ${ }^{(41)}$ & $13(\%)$ & $\begin{array}{l}\text { Amateur team-sport } \\
\text { participants }\end{array}$ & & - & $\mathrm{BJ} / 70$ & 6.0 & $\begin{array}{l}\text { BJ negligible nitrate } \\
\text { content }\end{array}$ & 1 & 180 & $\begin{array}{l}3 \text { sessions of } \\
6 \times 20 \mathrm{~m} \text { sprints }\end{array}$ & $\begin{array}{c}\text { Total sprint } \\
\text { time (s) }\end{array}$ & $\begin{array}{l}\mathrm{S}=69 \cdot 8(\mathrm{SD} 4 \cdot 9) \\
\mathrm{NS}=69 \cdot 9(\mathrm{SD} 4 \cdot 1) \\
\mathrm{D}=\mathrm{no}\end{array}$ \\
\hline $\begin{array}{l}\text { Christensen } \\
\text { et al. (1) }\end{array}$ & $8\left(0^{*}\right)$ & Recreationally active & 46 & 3 & $\mathrm{BJ} / 150$ & 9 & $\begin{array}{l}\text { Blackcurrant citrus } \\
\text { with } 0.2 \mathrm{mmol} \\
\text { nitrate }\end{array}$ & 1 & $180-249$ & Incremental leg exercise & $\begin{array}{l}\text { Peak power } \\
\text { output (W) }\end{array}$ & $\begin{array}{l}S=304(\mathrm{SD} 34) \\
N S=310(\mathrm{sD} 47) \\
\mathrm{D}=\mathrm{no}\end{array}$ \\
\hline $\begin{array}{l}\text { Christensen } \\
\quad \text { et al. (2) }\end{array}$ & $8\left({ }^{+}\right)$ & Recreationally active & 46 & 3 & $\mathrm{BJ} / 150$ & 9 & $\begin{array}{l}\text { Blackcurrant citrus } \\
\text { with } 0.2 \mathrm{mmol} \\
\text { nitrate }\end{array}$ & 1 & $180-249$ & $\begin{array}{l}\text { Incremental arm } \\
\text { exercise }\end{array}$ & $\begin{array}{l}\text { Peak power } \\
\text { output (W) }\end{array}$ & $\begin{array}{l}S=121(\mathrm{SD} 13) \\
N S=117(\mathrm{SD} 14) \\
\mathrm{D}=\mathrm{no}\end{array}$ \\
\hline$\underset{(1)^{(43)}}{\operatorname{Coggan}}$ et al. & $\begin{array}{l}12(7 \text { क } \\
\text { and } \\
5 \text { q) }\end{array}$ & Healthy & & - & $\mathrm{BJ} / 140$ & $11 \cdot 2$ & $\begin{array}{l}\text { BJ negligible nitrate } \\
\text { content }\end{array}$ & 1 & 120 & $\begin{array}{l}\text { Knee extensor } \\
\text { contractile function } \\
(1.57 \mathrm{rad} / \mathrm{s})\end{array}$ & $\begin{array}{l}\text { Peak power } \\
\text { output } \\
\text { (W/kg) }\end{array}$ & $\begin{array}{l}S=3.31(\text { SD } 0.55) \\
N S=3.38(\text { SD } 0.72) \\
D=n o\end{array}$ \\
\hline $\begin{array}{l}\text { Coggan } \\
\text { et al. }(2)^{(43)}\end{array}$ & $\begin{array}{l}12(7)^{+} \\
\text {and } \\
5 \text { q) }\end{array}$ & Healthy & & - & $\mathrm{BJ} / 140$ & $11 \cdot 2$ & $\begin{array}{l}\text { BJ negligible nitrate } \\
\text { content }\end{array}$ & 1 & 120 & $\begin{array}{l}\text { Knee extensor } \\
\text { contractile function } \\
(3.14 \mathrm{rad} / \mathrm{s})\end{array}$ & $\begin{array}{l}\text { Peak power } \\
\text { output } \\
\text { (W/kg) }\end{array}$ & $\begin{array}{l}S=5 \cdot 38(\text { SD 1.10) } \\
N S=5 \cdot 48(\text { SD 1.31) } \\
D=n o\end{array}$ \\
\hline $\begin{array}{l}\text { Coggan } \\
\text { et al. }(3)^{(43)}\end{array}$ & $\begin{array}{l}12\left(70^{+}\right. \\
\text {and } \\
5 \text { \%) }\end{array}$ & Healthy & & - & $\mathrm{BJ} / 140$ & $11 \cdot 2$ & $\begin{array}{l}\text { BJ negligible nitrate } \\
\text { content }\end{array}$ & 1 & 120 & $\begin{array}{l}\text { Knee extensor } \\
\text { contractile function } \\
(4.17 \mathrm{rad} / \mathrm{s})\end{array}$ & $\begin{array}{l}\text { Peak power } \\
\text { output } \\
\text { (W/kg) }\end{array}$ & $\begin{array}{l}S=6.76(\text { sD } 1.59) \\
N S=6.67 \text { (sD 1.73) } \\
D=\text { no }\end{array}$ \\
\hline$\underset{\text { et al. }(4)^{(43)}}{\text { Coggan }}$ & $\begin{array}{l}12\left(70^{+}\right. \\
\text {and } \\
5 \text { p) }\end{array}$ & Healthy & & - & $\mathrm{BJ} / 140$ & $11 \cdot 2$ & $\begin{array}{l}\text { BJ negligible nitrate } \\
\text { content }\end{array}$ & 1 & 120 & $\begin{array}{l}\text { Knee extensor } \\
\text { contractile function } \\
(6.28 \mathrm{rad} / \mathrm{s})\end{array}$ & $\begin{array}{l}\text { Peak power } \\
\text { output } \\
\text { (W/kg) }\end{array}$ & $\begin{array}{l}S=7.64(\text { SD } 1.80) \\
N S=7.34(\text { sD } 1.87) \\
D=\text { yes }\end{array}$ \\
\hline $\begin{array}{l}\text { Corry } \\
\quad \text { et al. }\end{array}$ & $10\left(0^{+}\right)$ & Recreationally active & & - & $\mathrm{BJ} / 140$ & 8.0 & $\begin{array}{l}\text { Low-energy } \\
\text { blackcurrant juice } \\
\text { with negligible } \\
\mathrm{NO}_{3}^{-}\end{array}$ & 2 & 40 & Wingate test & $\begin{array}{l}\text { Mean power } \\
\text { output } \\
(\text { W/kg) }\end{array}$ & $\begin{array}{l}S=7.95(\mathrm{sD} 0.55) \\
N S=7.63(\mathrm{sD} 0.91) \\
D=\text { no }\end{array}$ \\
\hline $\begin{array}{l}\text { Fulford } \\
\text { et al. (1) }\end{array}$ & $8\left({ }^{+}\right)$ & $\begin{array}{l}\text { Healthy, physically } \\
\text { active }\end{array}$ & & - & $\mathrm{BJ} / 250$ & $10 \cdot 2$ & $\begin{array}{l}\text { BJ negligible nitrate } \\
\text { content }\end{array}$ & 1 & 150 & $\begin{array}{l}\text { Isometric maximum } \\
\text { voluntary contraction } \\
\text { protocol }\end{array}$ & $\begin{array}{l}\text { Mean force } \\
\text { of peak } \\
\text { contraction }(\mathrm{N})\end{array}$ & $\begin{array}{l}S=368(\mathrm{sD} 90) \\
N S=382(\mathrm{sD} 143) \\
\mathrm{D}=\mathrm{no}\end{array}$ \\
\hline $\begin{array}{l}\text { Fulford et al. } \\
\qquad(2)^{(45)}\end{array}$ & $8\left({ }^{3}\right)$ & $\begin{array}{l}\text { Healthy, physically } \\
\text { active }\end{array}$ & & - & $\mathrm{BJ} / 250$ & $10 \cdot 2$ & $\begin{array}{l}\text { BJ negligible nitrate } \\
\text { content }\end{array}$ & $5(2 \times / d)$ & 150 & $\begin{array}{l}\text { Isometric maximum } \\
\text { voluntary contraction } \\
\text { protocol }\end{array}$ & $\begin{array}{l}\text { Mean force } \\
\quad \text { of peak } \\
\text { contraction (N) }\end{array}$ & $\begin{array}{l}S=380(\text { SD } 65) \\
N S=387(\text { SD 119) } \\
D=n o\end{array}$ \\
\hline $\begin{array}{l}\text { Fulford et al. } \\
(3)^{(45)}\end{array}$ & $8\left({ }^{*}\right)$ & $\begin{array}{l}\text { Healthy, physically } \\
\text { active }\end{array}$ & & - & $\mathrm{BJ} / 250$ & $10 \cdot 2$ & $\begin{array}{l}\text { BJ negligible nitrate } \\
\text { content }\end{array}$ & $15(2 \times / d)$ & 150 & $\begin{array}{l}\text { Isometric maximum } \\
\text { voluntary contraction } \\
\text { protocol }\end{array}$ & $\begin{array}{l}\text { Mean force } \\
\text { of peak } \\
\text { contraction (N) }\end{array}$ & $\begin{array}{l}S=408(\mathrm{sD} 110) \\
\mathrm{NS}=365(\mathrm{sD} 115) \\
\mathrm{D}=\mathrm{no}\end{array}$ \\
\hline
\end{tabular}




\section{W British Journal of Nutrition}

\begin{tabular}{|c|c|c|c|c|c|c|c|c|c|c|c|c|}
\hline \multirow[b]{3}{*}{ References } & \multirow{3}{*}{$\begin{array}{l}\text { No. of } \\
\text { subjects } \\
\left({ }^{\circ}, \text { P) }\right.\end{array}$} & \multirow{3}{*}{$\begin{array}{l}\text { Characteristics of } \\
\text { subjects }\end{array}$} & \multicolumn{7}{|c|}{ Nitrate supplementation } & \multirow{3}{*}{ Exercise protocol } & \multirow{3}{*}{$\begin{array}{l}\text { Variable of } \\
\text { physical } \\
\text { performance }\end{array}$} & \multirow[b]{3}{*}{ Results } \\
\hline & & & \multicolumn{2}{|c|}{$\begin{array}{l}\mathrm{VO}_{2 \text { peak }} / \mathrm{NO}_{2 \max } \\
\text { (ml/kg per min) }\end{array}$} & \multirow{2}{*}{$\begin{array}{l}\text { Ingested fluid/ } \\
\text { volume (ml) }\end{array}$} & \multirow{2}{*}{$\begin{array}{l}\text { Dose } \\
(\mathrm{mmol})\end{array}$} & \multirow[b]{2}{*}{ Placebo substance } & \multirow{2}{*}{$\begin{array}{l}\text { Days of } \\
\text { supplementation }\end{array}$} & \multirow{2}{*}{$\begin{array}{l}\text { Time before } \\
\text { trial (min) }\end{array}$} & & & \\
\hline & & & Mean & SD & & & & & & & & \\
\hline $\begin{array}{l}\text { Kelly et al. } \\
\text { (1) }\end{array}$ & $9\left(\delta^{*}\right)$ & Recreationally active & 54.5 & 7.5 & BJ/500 (250 + 250) & $8 \cdot 2$ & $\begin{array}{l}\text { BJ negligible nitrate } \\
\text { content }\end{array}$ & $7-12$ & 150 & $\begin{array}{l}\text { Severe-intensity } \\
\text { exercise ( } 60 \% \text { peak } \\
\text { power output) }\end{array}$ & Time (s) & $\begin{array}{l}S=696(\text { SD } 120) \\
N S=593(\text { (sD 68) } \\
D=\text { yes }\end{array}$ \\
\hline $\begin{array}{l}\text { Kelly et al. } \\
\text { (2) }\end{array}$ & $9\left(0^{7}\right)$ & Recreationally active & 54.5 & 7.5 & $\mathrm{BJ} / 500(250+250)$ & $8 \cdot 2$ & $\begin{array}{l}\text { BJ negligible nitrate } \\
\text { content }\end{array}$ & $7-12$ & 150 & $\begin{array}{l}\text { Severe-intensity } \\
\text { exercise (70\% peak } \\
\text { power output) }\end{array}$ & Time (s) & $\begin{array}{l}S=452(\text { SD } 106) \\
N S=390(\text { SD } 86) \\
D=\text { yes }\end{array}$ \\
\hline $\begin{array}{l}\text { Kelly et al. } \\
(3)^{(46)}\end{array}$ & $9\left(\delta^{\top}\right)$ & Recreationally active & $54 \cdot 5$ & 7.5 & $\mathrm{BJ} / 500(250+250)$ & $8 \cdot 2$ & $\begin{array}{l}\text { BJ negligible nitrate } \\
\text { content }\end{array}$ & $7-12$ & 150 & $\begin{array}{l}\text { Severe-intensity } \\
\text { exercise }(80 \% \text { peak } \\
\text { power output) }\end{array}$ & Time (s) & $\begin{array}{l}S=294(\mathrm{sD} 50) \\
\mathrm{NS}=263(\mathrm{sD} 50) \\
\mathrm{D}=\text { yes }\end{array}$ \\
\hline $\begin{array}{l}\text { Kelly et al. } \\
\text { (4) }\end{array}$ & $9\left({ }^{(}\right)$ & Recreationally active & 54.5 & 7.5 & $\mathrm{BJ} / 500(250+250)$ & 8.2 & $\begin{array}{l}\text { BJ negligible nitrate } \\
\text { content }\end{array}$ & $7-12$ & 150 & $\begin{array}{l}\text { Severe-intensity } \\
\text { exercise (100\% peak } \\
\text { power output) }\end{array}$ & Time (s) & $\begin{array}{l}S=182(S D ~ 37) \\
N S=166(S D 26) \\
D=n o\end{array}$ \\
\hline $\begin{array}{l}\text { Kokkinoplitis } \\
\text { and } \\
\text { Chester } \\
\text { (47) }\end{array}$ & $7\left({ }^{(}\right)$ & Healthy & - & - & $\mathrm{BJ} / 70$ & 6.4 & Blackcurrant juice & 1 & 180 & $\begin{array}{l}\text { Repeated high-intensity } \\
\text { sprints }(5 \times 6 \mathrm{~s})\end{array}$ & $\begin{array}{l}\text { Mean peak } \\
\text { power } \\
\text { output (W) }\end{array}$ & $\begin{array}{l}\mathrm{S}=4133.5(\mathrm{SD} 674.4) \\
\mathrm{NS}=3938.3 \\
(\mathrm{SD} 603.1) \\
D=\text { no }\end{array}$ \\
\hline $\begin{array}{l}\text { Lansley }_{\text {et al. }}^{(23)}\end{array}$ & $9($ ( ) & Physically active & 55 & 7 & $\mathrm{BJ} / 500$ & $6 \cdot 2$ & $\begin{array}{l}\text { BJ negligible nitrate } \\
\text { content }\end{array}$ & 6 & 180 & Severe-intensity running & Time (min) & $\begin{array}{l}S=8.7(\text { SD } 1.8) \\
N S=7.6(\text { SD } 1.5) \\
D=\text { yes }\end{array}$ \\
\hline $\begin{array}{l}\text { Larsen } \\
\quad \text { et al. }\end{array}$ & $\begin{array}{l}9(7 \text { o } \\
\text { and } \\
2 \text { o) }\end{array}$ & Healthy & 3.72 & $0.33^{*}$ & Sodium nitrate & $\begin{array}{l}0.033 \mathrm{mmol} / \mathrm{kg} \\
\text { body mass }\end{array}$ & Sodium chloride & $2(3 \times / d)$ & 40 & $\begin{array}{l}\text { Incremental exercise on } \\
\text { ergometers }\end{array}$ & Time (s) & $\begin{array}{l}S=563(\mathrm{sD} 90) \\
N S=524(\mathrm{SD} 93) \\
D=\text { no }\end{array}$ \\
\hline $\begin{array}{l}\text { Murphy } \\
\text { et al. }\end{array}$ & $\begin{array}{l}11\left(5 o^{+}\right. \\
\text {and } \\
6 \text { 9) }\end{array}$ & Recreationally fit & - & - & Baked beetroot & 8.0 & Cranberry relish & 1 & 60 & Time trial $5 \mathrm{~km}$ & $\begin{array}{l}\text { Running } \\
\text { speed }(\mathrm{km} / \mathrm{h})\end{array}$ & $\begin{array}{l}S=12.3(\text { SD } 9.0) \\
N S=11.9(\text { SD } 8.6) \\
D=\text { no }\end{array}$ \\
\hline $\begin{array}{l}\text { Nyakayiru } \\
\text { et al. }{ }^{(50)}\end{array}$ & $32\left(0^{*}\right)$ & Soccer players & & - & $\mathrm{BJ} / 140$ & $12 \cdot 9$ & $\begin{array}{l}\text { BJ negligible nitrate } \\
\text { content }\end{array}$ & 6 & 240 & Yo-Yo test & Distance $(\mathrm{m})$ & $\begin{array}{l}S=1623(\mathrm{sD} 48) \\
N S=1574(\mathrm{SD} 47) \\
D=\text { yes }\end{array}$ \\
\hline $\begin{array}{l}\text { Porcelli et al. } \\
(1)^{(51)}\end{array}$ & $8(ठ)$ & $\begin{array}{l}\text { Healthy individuals } \\
\text { with a low aerobic }\end{array}$ & $28 \cdot 2-$ & $-44 \cdot 1$ & Sodium nitrate & 5.5 & Sodium chloride & 6 & 210 & Time trial $3 \mathrm{~km}$ & Time (s) & $\begin{array}{l}S=886(\text { SD } 74) \\
N S=910(\text { SD } 82) \\
D=\text { yes }\end{array}$ \\
\hline $\begin{array}{l}\text { Porcelli et al. } \\
\text { (2) }\end{array}$ & $7($ () & $\begin{array}{l}\text { Healthy individuals } \\
\text { with a moderate } \\
\text { aerobic capacity }\end{array}$ & $45 \cdot 5-$ & -57.1 & Sodium nitrate & 5.5 & Sodium chloride & 6 & 210 & Time trial $3 \mathrm{~km}$ & Time (s) & $\begin{array}{l}S=723(\mathrm{sD} 90) \\
N S=734(\mathrm{SD} 93) \\
D=\text { yes }\end{array}$ \\
\hline $\begin{array}{l}\text { Porcelli et al. } \\
\text { (3) }\end{array}$ & $6\left(0^{*}\right)$ & $\begin{array}{l}\text { Healthy individuals } \\
\text { with a high aerobic } \\
\text { capacity }\end{array}$ & $63.9-$ & -81.7 & Sodium nitrate & $5 \cdot 5$ & Sodium chloride & 6 & 210 & Time trial $3 \mathrm{~km}$ & Time (s) & $\begin{array}{l}\mathrm{S}=627(\mathrm{sD} 30) \\
\mathrm{NS}=629(\mathrm{SD} 28) \\
\mathrm{D}=\mathrm{no}\end{array}$ \\
\hline $\begin{array}{l}\text { Rienks } \\
\quad \text { et al. }\end{array}$ & 10 (\$) & Healthy & $37 \cdot 1$ & $5 \cdot 3$ & $\mathrm{BJ} / 140$ & $12 \cdot 9$ & $\begin{array}{l}\text { BJ negligible nitrate } \\
\text { content }\end{array}$ & 1 & 150 & $\begin{array}{l}20 \text { min of cycling } \\
\text { exercise at RPE } 13\end{array}$ & $\begin{array}{l}\text { Total mechanical } \\
\text { work (kJ) }\end{array}$ & $\begin{array}{l}S=30 \cdot 3(\text { SD 5.3) } \\
N S=29 \cdot 8(\text { SD 6.1) } \\
D=n o\end{array}$ \\
\hline $\begin{array}{l}\text { Thompson } \\
\text { et al. }\end{array}$ & $16($ §) & $\begin{array}{l}\text { Healthy, recreationally } \\
\text { active }\end{array}$ & $47 \cdot 3$ & $6 \cdot 3$ & $\mathrm{BJ} / 500$ & $5 \cdot 0$ & $\begin{array}{l}\text { BJ negligible nitrate } \\
\text { content }\end{array}$ & 1 & 90 & $\begin{array}{l}\text { Continuous cycle } \\
\text { exercise test until } \\
\text { volitional exhaustion }\end{array}$ & $\begin{array}{l}\text { Exercise } \\
\text { tolerance (s) }\end{array}$ & $\begin{array}{l}S=185(\text { SD 122) } \\
N S=160 \text { (SD 109) } \\
D=\text { yes }\end{array}$ \\
\hline $\begin{array}{l}\text { Thompson } \\
\text { et al. }\end{array}$ & $16($ ( $)$ & $\begin{array}{l}\text { Recreational team- } \\
\text { sport players }\end{array}$ & 50 & 7 & $\mathrm{BJ} / 70$ & $6 \cdot 4$ & $\begin{array}{l}\text { BJ negligible nitrate } \\
\text { content }\end{array}$ & $7(2 \times / d)$ & 150 & Intermittent-sprint test & $\begin{array}{l}\text { Total work done } \\
\text { during the } \\
\text { sprints }(\mathrm{kJ})\end{array}$ & $\begin{array}{l}S=123(\mathrm{SD} 19) \\
\mathrm{NS}=119(\mathrm{sD} 17) \\
D=\text { ves }\end{array}$ \\
\hline $\begin{array}{l}\text { Vasconcellos } \\
\text { et al. }\end{array}$ & $\begin{array}{l}25(14 \text { o } \\
\text { and } \\
11 \text { \%) }\end{array}$ & Healthy & $\begin{array}{l}\delta=64 \cdot 31 \\
\wp=52 \cdot 79\end{array}$ & $\begin{array}{l}\delta=4.71 \\
q=4.57\end{array}$ & $\begin{array}{l}\text { Two beetroot gels with } \\
50 \mathrm{~g} \text { each and } \\
300 \mathrm{ml} \text { of water }\end{array}$ & 9.92 (SD 1.97) & Placebo gel & 1 & 90 & Severe-intensity running & Time (s) & $\begin{array}{l}S=395.4(\text { SD 179.6) } \\
N S=390.9(\text { SD 158.5) } \\
D=\text { no }\end{array}$ \\
\hline
\end{tabular}




\section{NS British Journal of Nutrition}

Table 1. Continued

\begin{tabular}{|c|c|c|c|c|c|c|c|c|c|c|c|c|}
\hline \multirow[b]{3}{*}{ References } & \multirow{3}{*}{$\begin{array}{l}\text { No. of } \\
\text { subjects } \\
\left(\delta^{\prime}, \text { }\right)\end{array}$} & \multirow{3}{*}{$\begin{array}{l}\text { Characteristics of } \\
\text { subjects }\end{array}$} & \multicolumn{7}{|c|}{ Nitrate supplementation } & \multirow[b]{3}{*}{ Exercise protocol } & \multirow{3}{*}{$\begin{array}{l}\text { Variable of } \\
\text { physical } \\
\text { performance }\end{array}$} & \multirow[b]{3}{*}{ Results } \\
\hline & & & \multicolumn{2}{|c|}{$\begin{array}{l}\mathrm{VO}_{2 \text { peak }} / \mathrm{NO}_{2 \max } \\
(\mathrm{ml} / \mathrm{kg} \text { per min) }\end{array}$} & \multirow{2}{*}{$\begin{array}{l}\text { Ingested fluid/ } \\
\text { volume (ml) }\end{array}$} & \multirow[b]{2}{*}{$\begin{array}{l}\text { Dose } \\
(\mathrm{mmol})\end{array}$} & \multirow[b]{2}{*}{ Placebo substance } & \multirow{2}{*}{$\begin{array}{l}\text { Days of } \\
\text { supplementation }\end{array}$} & \multirow{2}{*}{$\begin{array}{l}\text { Time before } \\
\text { trial (min) }\end{array}$} & & & \\
\hline & & & Mean & SD & & & & & & & & \\
\hline $\begin{array}{l}\text { Wylie et al. } \\
(1)^{(17)}\end{array}$ & $10\left(0^{\pi}\right)$ & $\begin{array}{l}\text { Healthy, recreationally } \\
\text { active }\end{array}$ & & & $\mathrm{BJ} / 70$ & 4.2 & Water & 1 & 150 & $\begin{array}{l}\text { Severe-intensity cycling } \\
\text { exercise }\end{array}$ & Time (s) & $\begin{array}{l}S=508(\text { SD } 102) \\
N S=470(\text { SD } 81) \\
D=n o\end{array}$ \\
\hline $\begin{array}{l}\text { Wylie et al. } \\
(2)^{(17)}\end{array}$ & $10\left(\delta^{\star}\right)$ & $\begin{array}{l}\text { Healthy, recreationally } \\
\text { active }\end{array}$ & & & $\mathrm{BJ} / 140$ & $8 \cdot 4$ & Water & 1 & 150 & $\begin{array}{l}\text { Severe-intensity cycling } \\
\text { exercise }\end{array}$ & Time (s) & $\begin{array}{l}S=570(\text { sD 153) } \\
\text { NS }=498(\text { SD 113) } \\
D=\text { ves }\end{array}$ \\
\hline $\begin{array}{l}\text { Wylie et al. } \\
(3)^{(17)}\end{array}$ & $10\left(\delta^{\pi}\right)$ & $\begin{array}{l}\text { Healthy, recreationally } \\
\text { active }\end{array}$ & & & $\mathrm{BJ} / 280$ & $12 \cdot 8$ & Water & 1 & 150 & $\begin{array}{l}\text { Severe-intensity cycling } \\
\text { exercise }\end{array}$ & Time (s) & $\begin{array}{l}S=552(\mathrm{sD} 117) \\
\mathrm{NS}=493(\mathrm{SD} 114)\end{array}$ \\
\hline Wylie et al. ${ }^{(56)}$ & $14(\overbrace{}^{\star})$ & $\begin{array}{l}\text { Recreational } \\
\text { team-sport players }\end{array}$ & 52 & 7 & $\mathrm{BJ} / 140$ & $4 \cdot 1$ & $\begin{array}{l}\text { BJ negligible nitrate } \\
\text { content }\end{array}$ & 2 & 150 & Yo-Yo IR1 & $\begin{array}{l}\text { Distance covered } \\
\quad(\mathrm{m})\end{array}$ & $\begin{array}{l}D=\text { yes } \\
S=1704(\mathrm{sD} 304) \\
N S=1636(\mathrm{SD} 288) \\
D=\text { yes }\end{array}$ \\
\hline $\begin{array}{l}\text { Wylie et al. } \\
(1)^{(57)}\end{array}$ & $10(\overbrace{}^{\lambda})$ & $\begin{array}{l}\text { Recreational } \\
\text { team-sport players }\end{array}$ & 58 & 8 & $\mathrm{BJ} / 140$ & $8 \cdot 2$ & $\begin{array}{l}\text { BJ negligible nitrate } \\
\text { content }\end{array}$ & 3 & 150 & $\begin{array}{l}\text { Maximal efforts }(24 \times 6-\mathrm{s} \\
\text { protocol) }\end{array}$ & $\begin{array}{l}\text { Mean power } \\
\text { output (W) }\end{array}$ & $\begin{array}{l}S=568(\text { sD 136) } \\
N S=539(\text { SD 136) } \\
D=\text { yes }\end{array}$ \\
\hline $\begin{array}{l}\text { Wylie et al. } \\
\text { (2) }\end{array}$ & $10\left(0^{7}\right)$ & $\begin{array}{l}\text { Recreational team- } \\
\text { sport players }\end{array}$ & 58 & 8 & $\mathrm{BJ} / 140$ & 8.2 & $\begin{array}{l}\text { BJ negligible nitrate } \\
\text { content }\end{array}$ & 4 & 150 & $\begin{array}{l}\text { Maximal efforts }(7 \times 30-\mathrm{s} \\
\text { protocol) }\end{array}$ & $\begin{array}{l}\text { Mean power } \\
\text { output (W) }\end{array}$ & $\begin{array}{l}S=558(\text { sD } 95) \\
N S=562(\text { sD } 94) \\
D=\text { no }\end{array}$ \\
\hline $\begin{array}{l}\text { Wylie et al. } \\
(3)^{(57)}\end{array}$ & $10\left(\delta^{\pi}\right)$ & $\begin{array}{l}\text { Recreational team- } \\
\text { sport players }\end{array}$ & 58 & 8 & $\mathrm{BJ} / 140$ & $8 \cdot 2$ & $\begin{array}{l}\text { BJ negligible nitrate } \\
\text { content }\end{array}$ & 5 & 150 & $\begin{array}{l}\text { Maximal efforts }(6 \times 60-\mathrm{s} \\
\text { protocol) }\end{array}$ & $\begin{array}{l}\text { Mean power } \\
\text { output (W) }\end{array}$ & $\begin{array}{l}S=374(\text { sD 57) } \\
N S=375(\text { SD 59) } \\
D=\text { no }\end{array}$ \\
\hline
\end{tabular}

T. Male; o, female; BJ, beetroot juice; NR, not reported; S, supplemented; NS, no supplementation; D, statistical difference.

*Absolute $\mathrm{VO}_{2}$ data in $\mathrm{l} / \mathrm{min}$ 


\section{Nesitish Journal of Nutrition}

Table 2. Study characteristics - athletes

(Mean values and standard deviations)

\begin{tabular}{|c|c|c|c|c|c|c|c|c|c|c|c|c|}
\hline \multirow[b]{3}{*}{ References } & \multirow{3}{*}{$\begin{array}{l}\text { No. of } \\
\text { subjects } \\
\left(\delta^{\prime}, \text { P) }\right.\end{array}$} & \multirow[b]{3}{*}{ Characteristics of subjects } & \multicolumn{7}{|c|}{ Nitrate supplementation } & \multirow[b]{3}{*}{ Exercise protocol } & \multirow{3}{*}{$\begin{array}{l}\text { Measure of } \\
\text { physical } \\
\text { performance }\end{array}$} & \multirow[b]{3}{*}{ Results } \\
\hline & & & \multicolumn{2}{|c|}{$\begin{array}{c}\mathrm{VO}_{2 \text { peak }} / \\
\mathrm{VO}_{2 \max }(\mathrm{m} / \mathrm{ml} / \mathrm{kg} \\
\text { per min) } \\
\end{array}$} & \multirow{2}{*}{$\begin{array}{l}\text { Ingested fluid/ } \\
\text { volume (ml) }\end{array}$} & \multirow[b]{2}{*}{ Dose (mmol) } & \multirow[b]{2}{*}{ Placebo substance } & \multirow[b]{2}{*}{$\begin{array}{c}\text { Days of } \\
\text { supplementation }\end{array}$} & \multirow[b]{2}{*}{$\begin{array}{l}\text { Time before } \\
\text { trial (min) }\end{array}$} & & & \\
\hline & & & Mean & SD & & & & & & & & \\
\hline $\begin{array}{l}\text { Bescós } \\
\text { et al. }\end{array}$ & $11\left({ }^{\star}\right)$ & Cyclists and triathletes & 65.1 & 6.2 & Sodium nitrate/250 & 11.8 & Sodium chloride & 1 & 180 & Incremental exercise & Time (s) & $\begin{array}{l}S=416(\text { SD } 32) \\
N S=409(\text { SD } 27) \\
D=n o\end{array}$ \\
\hline $\begin{array}{l}\text { Bescós et al. } \\
(1)^{(25)}\end{array}$ & $13\left(0^{7}\right)$ & Cyclists and triathletes & & - & Sodium nitrate/250 & $11 \cdot 6$ & Sodium chloride & 3 & 180 & $\begin{array}{l}\text { Distance trial }(40 \mathrm{~min}) \\
\text { in cycle ergometer }\end{array}$ & Distance $(\mathrm{km})$ & $\begin{array}{l}S=26 \cdot 4(S D 1 \cdot 1) \\
N S=26 \cdot 3(S D 1 \cdot 2) \\
D=\text { no }\end{array}$ \\
\hline $\begin{array}{l}\text { Bescós et al. } \\
(2)^{(25)}\end{array}$ & $13\left(\delta^{\pi}\right)$ & Cyclists and triathletes & & - & Sodium nitrate/250 & $11 \cdot 6$ & Sodium chloride & 3 & 180 & $\begin{array}{l}\text { Distance trial }(40 \mathrm{~min}) \\
\text { in cycle ergometer }\end{array}$ & $\begin{array}{l}\text { Mean power } \\
\text { output (W) }\end{array}$ & $\begin{array}{l}S=258(\mathrm{SD} 28) \\
\mathrm{NS}=257 \cdot 3(\mathrm{SD} 28) \\
\mathrm{D}=\mathrm{nO}\end{array}$ \\
\hline Bond et al. ${ }^{(20)}$ & $14(\overbrace{}^{\star})$ & Rowers & & - & $\mathrm{BJ} / 500(250+250)$ & 5.0 & Blackcurrant juice & 6 & NR & $\begin{array}{l}6 \times 500 \mathrm{~m} \text { rowing }- \\
\text { ergometer repetitions } \\
\text { at maximal intensity }\end{array}$ & Time (s) & $\begin{array}{l}U=n o \\
S=89.4 \\
N S=90 \cdot 1 \\
D=\text { no }\end{array}$ \\
\hline $\begin{array}{l}\text { Boorsma et al. } \\
(1)^{(58)}\end{array}$ & $8\left(0^{\star}\right)$ & Distance runners & 80 & 5 & $\begin{array}{l}\text { BJ/210 (on the } \\
\text { test day) and } 140 \\
\text { (other days) }\end{array}$ & $19 \cdot 5$ & $\begin{array}{l}\text { BJ negligible nitrate } \\
\text { content }\end{array}$ & 1 & 150 & Time trial $1500 \mathrm{~m}$ & Time (s) & $\begin{array}{l}S=250.7(\mathrm{sD} 4 \cdot 3) \\
N S=250 \cdot 4(\mathrm{sD} 7 \cdot 0) \\
D=\text { no }\end{array}$ \\
\hline $\begin{array}{l}\text { Boorsma et al. } \\
(2)^{(58)}\end{array}$ & $8\left(0^{\star}\right)$ & Distance runners & 80 & 5 & $\begin{array}{l}\text { BJ/210 (on the } \\
\text { test day) and } 140 \\
\text { (other days) }\end{array}$ & $\begin{array}{l}19.5 \text { (on the } \\
\text { test day) and } 13 \\
\text { (other days) }\end{array}$ & $\begin{array}{l}\text { BJ negligible nitrate } \\
\text { content }\end{array}$ & 8 & 150 & Time trial $1500 \mathrm{~m}$ & Time (s) & $\begin{array}{l}S=250.5(\text { SD } 6.2) \\
N S=251.4(\text { SD } 7.6) \\
D=n o\end{array}$ \\
\hline $\begin{array}{l}\text { Callahan et al. } \\
(1)^{(59)}\end{array}$ & $8\left({ }^{\top}\right)$ & Endurance-trained cyclists & $65 \cdot 2$ & $4 \cdot 2$ & $\begin{array}{l}\text { Gelatine } \\
\text { capsules + water } \\
(400 \mathrm{ml})\end{array}$ & 5.0 & $\begin{array}{l}\text { Gelatine capsules }(90 \% \\
\text { BeetEssence and } \\
10 \% \text { Black Cherry } \\
\text { cool-aid) }\end{array}$ & 3 & 60 & Time trial $4000 \mathrm{~m}$ & $\begin{array}{l}\text { Mean power } \\
\text { output (W) }\end{array}$ & $\begin{array}{l}S=388(\mathrm{sD} 54) \\
N S=386(\mathrm{sD} 56) \\
D=\text { no }\end{array}$ \\
\hline $\begin{array}{l}\text { Callahan et al. } \\
(2)^{(59)}\end{array}$ & $8\left(0^{7}\right)$ & Endurance-trained cyclists & $65 \cdot 2$ & $4 \cdot 2$ & $\begin{array}{l}\text { Gelatine } \\
\text { capsules + water } \\
(400 \mathrm{ml})\end{array}$ & $5 \cdot 0$ & $\begin{array}{l}\text { Gelatine capsules (90\% } \\
\text { Beet Essence and } \\
10 \% \text { Black Cherry } \\
\text { cool-aid) }\end{array}$ & 3 & 60 & Time trial $4000 \mathrm{~m}$ & Time (s) & $\begin{array}{l}S=337.4(S D 17 \cdot 1) \\
N S=338 \cdot 1(S D \\
18 \cdot 0) \\
D=n o\end{array}$ \\
\hline $\begin{array}{c}\text { Cermak et al. } \\
(1)^{(21)}\end{array}$ & $12\left(0^{\pi}\right)$ & Cyclists and triathletes & 58 & 2 & $\mathrm{BJ} / 140(70+70)$ & 8.0 & $\begin{array}{l}\text { BJ negligible nitrate } \\
\text { content }\end{array}$ & 6 & 150 & Time trial $10 \mathrm{~km}$ & Time (s) & $\begin{array}{l}S=953(\mathrm{sD} 72 \cdot 5) \\
\mathrm{NS}=965(\mathrm{sD} 72 \cdot 5) \\
\mathrm{D}=\text { yes }\end{array}$ \\
\hline$\underset{(2)^{(21)}}{\text { Cermak al al. }}$ & $12\left({ }^{\star}\right)$ & Cyclists and triathletes & 58 & 2 & $\mathrm{BJ} / 140(70+70)$ & $8 \cdot 0$ & $\begin{array}{l}\text { BJ negligible nitrate } \\
\text { content }\end{array}$ & 6 & 150 & Time trial $10 \mathrm{~km}$ & $\begin{array}{l}\text { Mean power } \\
\text { output (W) }\end{array}$ & $\begin{array}{l}S=294(\mathrm{SD} 41 \cdot 5) \\
\mathrm{NS}=288(\mathrm{SD} 41 \cdot 5) \\
\mathrm{D}=\text { yes }\end{array}$ \\
\hline$\underset{(1)^{(27)}}{\text { Cermak }}$ et al. & $20\left(0^{x}\right)$ & Cyclists or triathletes & 60 & 1 & $\mathrm{BJ} / 140$ & $8 \cdot 7$ & $\begin{array}{l}\text { BJ negligible nitrate } \\
\text { content }\end{array}$ & 1 & 150 & $\begin{array}{l}\text { Time trial approximately } \\
1073 \mathrm{~kJ}\end{array}$ & Time $(\mathrm{min})$ & $\begin{array}{l}S=65.5(\mathrm{SD} 4 \cdot 8) \\
N S=65 \cdot 0(\mathrm{SD} 4 \cdot 8) \\
D=\text { no }\end{array}$ \\
\hline$\underset{(2)^{(27)}}{\text { Cermak et al. }}$ & $20\left(0^{\star}\right)$ & Cyclists or triathletes & 60 & 1 & $\mathrm{BJ} / 140$ & 8.7 & $\begin{array}{l}\text { BJ negligible nitrate } \\
\text { content }\end{array}$ & 1 & 150 & $\begin{array}{l}\text { Time trial approximately } \\
1073 \mathrm{~kJ}\end{array}$ & $\begin{array}{l}\text { Mean power } \\
\text { output (W) }\end{array}$ & $\begin{array}{l}S=275(\mathrm{sD} 30.9) \\
N S=278(\mathrm{SD} 30 \cdot 9) \\
D=n 0\end{array}$ \\
\hline $\begin{array}{l}\text { Christensen } \\
\text { et al. (1) }\end{array}$ & $10\left(0^{7}\right)$ & Cyclists & $72 \cdot 1$ & 4.5 & $\mathrm{BJ} / 500$ & 8.0 & $\begin{array}{l}\text { Apple and blackcurrant } \\
\text { juice }\end{array}$ & 4 & 180 & $\begin{array}{l}\text { Repeated sprint test } \\
\qquad(6,20 \mathrm{~s})\end{array}$ & $\begin{array}{l}\text { Mean power } \\
\text { output (W) }\end{array}$ & $\begin{array}{l}S=630(\mathrm{sD} 84) \\
N S=630(\mathrm{sD} 92) \\
D=n \mathrm{O}\end{array}$ \\
\hline $\begin{array}{l}\text { Christensen } \\
\text { et al. (2) }\end{array}$ & $10\left(0^{\star}\right)$ & Cyclists & $72 \cdot 1$ & 4.5 & $\mathrm{BJ} / 500$ & 8.0 & $\begin{array}{l}\text { Apple and blackcurrant } \\
\text { juice }\end{array}$ & 6 & 180 & $\begin{array}{l}\text { Time trial } 1677 \mathrm{~kJ} \\
\quad(400 \mathrm{kcal})\end{array}$ & Time $(\mathrm{min})$ & $\begin{array}{l}D=n o \\
S=18 \cdot 33 \\
N S=18 \cdot 61 \\
D=\text { no }\end{array}$ \\
\hline $\begin{array}{l}\text { Christensen } \\
\text { et al. }(3)^{(32)}\end{array}$ & $10\left(0^{\star}\right)$ & Cyclists & $72 \cdot 1$ & 4.5 & $\mathrm{BJ} / 500$ & 8.0 & $\begin{array}{l}\text { Apple and blackcurrant } \\
\text { juice }\end{array}$ & 6 & 180 & $\begin{array}{l}\text { Time trial } 1677 \mathrm{~kJ} \\
(400 \mathrm{kcal})\end{array}$ & $\begin{array}{l}\text { Mean power } \\
\text { output (W) }\end{array}$ & $\begin{array}{l}S=290(\mathrm{SD} 43 \\
\mathrm{NS}=285(\mathrm{SD} 44) \\
\mathrm{D}=\mathrm{no}\end{array}$ \\
\hline $\begin{array}{l}\text { Christensen } \\
\text { et al. (3) }\end{array}$ & $9\left({ }^{\star}\right)$ & Endurance-trained cyclists & 64 & 3 & $\mathrm{BJ} / 150$ & 9 & $\begin{array}{l}\text { Blackcurrant citrus with } \\
0.2 \mathrm{mmol} \text { nitrate }\end{array}$ & 1 & $180-249$ & Incremental leg exercise & $\begin{array}{l}\text { Peak power } \\
\text { output (W) }\end{array}$ & $\begin{array}{l}S=418(\mathrm{sD} 47) \\
\mathrm{NS}=406(\mathrm{sD} 46)\end{array}$ \\
\hline $\begin{array}{l}\text { Christensen } \\
\text { et al. (4) }\end{array}$ & $9\left(\delta^{7}\right)$ & Endurance-trained cyclists & 64 & 3 & $\mathrm{BJ} / 150$ & 9 & $\begin{array}{l}\text { Blackcurrant citrus with } \\
0.2 \mathrm{mmol} \text { nitrate }\end{array}$ & 1 & $180-249$ & Incremental arm exercise & $\begin{array}{l}\text { Peak power } \\
\text { output (W) }\end{array}$ & $\begin{array}{l}S=140(\text { SD 17) } \\
N S=141(\text { SD 20) }\end{array}$ \\
\hline $\begin{array}{l}\text { Glaister } \\
\text { et al. }\end{array}$ & 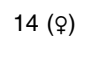 & Cyclists and triathletes & $52 \cdot 3$ & 4.9 & $\mathrm{BJ} / 70$ & $7 \cdot 3$ & $\begin{array}{l}\text { BJ negligible nitrate } \\
\text { content }\end{array}$ & 1 & 150 & Time trial $20 \mathrm{~km}$ & Time (min) & $\begin{array}{l}S=35 \cdot 3(\text { SD } 1 \cdot 5) \\
N S=35 \cdot 3(\text { SD } 1 \cdot 7) \\
D=n O\end{array}$ \\
\hline $\begin{array}{l}\text { Hoon et al. } \\
(1)^{(61)}\end{array}$ & $28\left(0^{7}\right)$ & Cyclists & & - & $\mathrm{BJ} / 70$ & $4 \cdot 1$ & $\begin{array}{l}\text { BJ negligible nitrate } \\
\text { content }\end{array}$ & 1 & 75 & Time trial $4 \mathrm{~min}$ & $\begin{array}{l}\text { Mean power } \\
\text { output (W) }\end{array}$ & $\begin{array}{l}\mathrm{D}=\mathrm{no} \\
\mathrm{S}=403(\mathrm{sD} 52) \\
\mathrm{NS}=396(\mathrm{SD} 57) \\
\mathrm{D}=\mathrm{no}\end{array}$ \\
\hline
\end{tabular}




\section{N's British Journal of Nutrition}

Nitrate supplementation

\begin{tabular}{|c|c|c|c|c|c|c|c|c|c|c|c|c|}
\hline \multirow[b]{3}{*}{ References } & \multirow{3}{*}{$\begin{array}{l}\text { No. of } \\
\text { subjects } \\
\left(\delta^{2}, \text { P) }\right.\end{array}$} & \multirow[b]{3}{*}{ Characteristics of subjects } & \multicolumn{7}{|c|}{ Nitrate supplementation } & \multirow[b]{3}{*}{ Exercise protocol } & \multirow{3}{*}{$\begin{array}{l}\text { Measure of } \\
\text { physical } \\
\text { performance }\end{array}$} & \multirow[b]{3}{*}{ Results } \\
\hline & & & \multicolumn{2}{|c|}{$\begin{array}{c}\mathrm{VO}_{2 \text { peak }} / \\
\mathrm{VO}_{2 \max }(\mathrm{m} / / \mathrm{kg} \\
\text { per min) } \\
\end{array}$} & \multirow{2}{*}{$\begin{array}{l}\text { Ingested fluid/ } \\
\text { volume (ml) }\end{array}$} & \multirow[b]{2}{*}{ Dose $(\mathrm{mmol})$} & \multirow[b]{2}{*}{ Placebo substance } & \multirow[b]{2}{*}{$\begin{array}{l}\text { Days of } \\
\text { supplementation }\end{array}$} & \multirow[b]{2}{*}{$\begin{array}{l}\text { Time before } \\
\text { trial (min) }\end{array}$} & & & \\
\hline & & & Mean & SD & & & & & & & & \\
\hline $\begin{array}{l}\text { Hoon et al. } \\
(2)^{(61)}\end{array}$ & $28\left(0^{7}\right)$ & Cyclists & & - & $\mathrm{BJ} / 70$ & 4.1 & $\begin{array}{l}\text { BJ negligible nitrate } \\
\text { content }\end{array}$ & 1 & 150 & Time trial $4 \mathrm{~min}$ & $\begin{array}{l}\text { Mean power } \\
\text { output (W) }\end{array}$ & $\begin{array}{l}S=402(\text { SD } 47 \\
N S=396(\text { SD } 57) \\
D=\text { no }\end{array}$ \\
\hline $\begin{array}{l}\text { Hoon et al. } \\
(1)^{(62)}\end{array}$ & $10\left(0^{7}\right)$ & Rowers & & - & $\mathrm{BJ} / 70$ & $4 \cdot 2$ & $\begin{array}{l}\text { BJ negligible nitrate } \\
\text { content }\end{array}$ & 1 & 120 & Time trial $2000 \mathrm{~m}$ & Time (s) & $\begin{array}{l}S=383.4(\text { sD } 8.7) \\
N S=383 \cdot 5(\text { sD } 9) \\
D=n o\end{array}$ \\
\hline $\begin{array}{l}\text { Hoon et al. } \\
(2)^{(62)}\end{array}$ & $10\left(0^{7}\right)$ & Rowers & & - & $\mathrm{BJ} / 140$ & $8 \cdot 4$ & $\begin{array}{l}\text { BJ negligible nitrate } \\
\text { content }\end{array}$ & 1 & 120 & Time trial $2000 \mathrm{~m}$ & Time (s) & $\begin{array}{l}S=381.9(\mathrm{SD} 9) \\
N S=383 \cdot 5(\mathrm{SD} 9) \\
D=\text { yes }\end{array}$ \\
\hline$\underset{(1)^{(63)}}{\operatorname{Kramer}}$ et al. & $12\left(0^{\star}\right)$ & CrossFit & 48.5 & $7 \cdot 0$ & Potassium nitrate & 8.0 & $\begin{array}{l}\text { Nitrate-free } \\
\text { potassium chloride }\end{array}$ & 6 & 1440 & Wingate test & Wingate peak & $\begin{array}{l}S=948.0(\mathrm{sD} 186.8) \\
\mathrm{NS}=905.0 \\
(\mathrm{SD} 157.2)\end{array}$ \\
\hline$\underset{(2)^{(63)}}{\text { Kramer et al. }}$ & $12\left(\delta^{\star}\right)$ & CrossFit & 48.5 & 7.0 & Potassium nitrate & 8.0 & $\begin{array}{l}\text { Nitrate-free } \\
\text { potassium chloride }\end{array}$ & 6 & 1440 & Time trial $2 \mathrm{~km}$ & Time (s) & $\begin{array}{l}S=459.7(\text { SD } 23.9) \\
N S=459.8 \\
(S D 24 \cdot 8) \\
D=\text { no }\end{array}$ \\
\hline $\begin{array}{l}\text { Lane et al. } \\
\quad(1)^{(64)}\end{array}$ & $12\left(0^{*}\right)$ & Cyclists and triathletes & 71.6 & 4.6 & $\mathrm{BJ} / 70$ & 8.4 & $\begin{array}{l}\text { BJ negligible nitrate } \\
\text { content }\end{array}$ & 2 & 130 & Time trial $43.83 \mathrm{~km}$ & Time (min) & $\begin{array}{l}\mathrm{S}=64 \cdot 0(\mathrm{sD} 2 \cdot 8) \\
\mathrm{NS}=63 \cdot 5(\mathrm{sD} 3 \cdot 2) \\
\mathrm{D}=\mathrm{no}\end{array}$ \\
\hline $\begin{array}{l}\text { Lane etal. } \\
(\text { (2) }\end{array}$ & $12\left(0^{7}\right)$ & Cyclists and triathletes & 71.6 & 4.6 & $\mathrm{BJ} / 70$ & 8.4 & $\begin{array}{l}\text { BJ negligible nitrate } \\
\text { content }\end{array}$ & 2 & 130 & Time trial $43.83 \mathrm{~km}$ & $\begin{array}{l}\text { Power output } \\
\text { (W) }\end{array}$ & $\begin{array}{l}S=298(\mathrm{SD} 35 \\
\mathrm{NS}=303(\mathrm{SD} 41) \\
\mathrm{D}=\mathrm{no}\end{array}$ \\
\hline $\begin{array}{l}\text { Lane et al. } \\
(3)^{(64)}\end{array}$ & 12 () & Cyclists and triathletes & 59.9 & $5 \cdot 1$ & $\mathrm{BJ} / 70$ & 8.4 & $\begin{array}{l}\text { BJ negligible nitrate } \\
\text { content }\end{array}$ & 2 & 130 & Time trial $29.35 \mathrm{~km}$ & Time (min) & $\begin{array}{l}S=51 \cdot 6(\text { SD } 2 \cdot 6) \\
N S=51 \cdot 6(\text { SD } 2 \cdot 5) \\
D=n o\end{array}$ \\
\hline $\begin{array}{l}\text { Lane et al. } \\
\quad(4)^{(64)}\end{array}$ & 12 (ㅇ) & Cyclists and triathletes & 59.9 & $5 \cdot 1$ & $\mathrm{BJ} / 70$ & 8.4 & $\begin{array}{l}\text { BJ negligible nitrate } \\
\text { content }\end{array}$ & 2 & 130 & Time trial $29.35 \mathrm{~km}$ & $\begin{array}{l}\text { Power output } \\
\text { (W) }\end{array}$ & $\begin{array}{l}S=207(\text { SD } 31 \\
N S=207(\text { sD 29) } \\
D=\text { no }\end{array}$ \\
\hline $\begin{array}{l}\text { Lansley et al. } \\
(1)^{(22)}\end{array}$ & $9\left(\delta^{\star}\right)$ & Cyclists & $56 \cdot 0$ & $5 \cdot 7$ & $\mathrm{BJ} / 500$ & $6 \cdot 2$ & $\begin{array}{l}\text { BJ negligible nitrate } \\
\text { content }\end{array}$ & 1 & 120 & Time trial $4 \mathrm{~km}$ & Time (min) & $\begin{array}{l}S=6.27(\mathrm{sD} 0.35) \\
N S=6.45(\mathrm{SD} 0.42) \\
D=\text { yes }\end{array}$ \\
\hline $\begin{array}{l}\text { Lansley et al. } \\
\text { (2) }\end{array}$ & $9\left({ }^{*}\right)$ & Cyclists & $56 \cdot 0$ & 5.7 & $\mathrm{BJ} / 500$ & $6 \cdot 2$ & $\begin{array}{l}\text { BJ negligible nitrate } \\
\text { content }\end{array}$ & 1 & 120 & Time trial $4 \mathrm{~km}$ & $\begin{array}{l}\text { Mean power } \\
\text { output (W) }\end{array}$ & $\begin{array}{l}S=292(\text { SD } 44) \\
N S=279(\text { SD } 51) \\
D=\text { yes }\end{array}$ \\
\hline $\begin{array}{l}\text { Lansley et al. } \\
(3)^{(22)}\end{array}$ & $9\left({ }^{(}\right)$ & Cyclists & $56 \cdot 0$ & 5.7 & $\mathrm{BJ} / 500$ & $6 \cdot 2$ & $\begin{array}{l}\text { BJ negligible nitrate } \\
\text { content }\end{array}$ & 1 & 120 & Time trial $16.1 \mathrm{~km}$ & Time $(\mathrm{min})$ & $\begin{array}{l}S=26 \cdot 9(\text { SD } 1.8) \\
N S=27.7(\text { SD } 2 \cdot 1) \\
D=\text { yes }\end{array}$ \\
\hline $\begin{array}{l}\text { Lansley et al. } \\
(4)^{(22)}\end{array}$ & $9\left({ }^{(}\right)$ & Cyclists & $56 \cdot 0$ & $5 \cdot 7$ & $\mathrm{BJ} / 500$ & $6 \cdot 2$ & $\begin{array}{l}\text { BJ negligible nitrate } \\
\text { content }\end{array}$ & 1 & 120 & Time trial $16.1 \mathrm{~km}$ & $\begin{array}{l}\text { Mean power } \\
\text { output (W) }\end{array}$ & $\begin{array}{l}S=247(\mathrm{sD} 44 \\
N S=233(\mathrm{sD} 43) \\
D=\text { yes }\end{array}$ \\
\hline $\begin{array}{l}\text { Lowings } \\
\text { et al. }\end{array}$ & $\begin{array}{l}10(50 \\
\text { and } \\
5 \text { q) }\end{array}$ & Swimmers & & - & $\mathrm{BJ} / 140(70+70)$ & 12.5 & $\begin{array}{l}\text { BJ negligible nitrate } \\
\text { content }\end{array}$ & 1 & 180 & $\begin{array}{l}\text { Swim time trial } \\
168 \mathrm{~m}\end{array}$ & Time (s) & $\begin{array}{l}S=10.3(\text { sD } 8.1) \\
N S=131 \cdot 5(\text { SD } 9.0) \\
D=\text { yes }\end{array}$ \\
\hline$\underset{(1)^{(66)}}{\text { Martin et al. }}$ & $\begin{array}{l}16\left(90^{+}\right. \\
\text {and } \\
7 \text { q) }\end{array}$ & Team-sport players & $47 \cdot 2$ & 8.5 & $\mathrm{BJ} / 70$ & 4.83 & $\begin{array}{l}\text { BJ negligible nitrate } \\
\text { content }\end{array}$ & 1 & 120 & $\begin{array}{l}\text { 8-s bouts of high-intensity } \\
\text { intermittent-sprint test }\end{array}$ & $\begin{array}{l}\text { No. of sprints } \\
\text { completed }\end{array}$ & $\begin{array}{l}S=13(\text { SD } 5) \\
N S=15(\text { sD } 6) \\
D=\text { yes }\end{array}$ \\
\hline $\begin{array}{l}\text { Martin et al. } \\
(2)^{(66)}\end{array}$ & $\begin{array}{l}16\left(90^{+}\right. \\
\text {and } \\
7 \%)\end{array}$ & Team-sport players & $47 \cdot 2$ & 8.5 & $\mathrm{BJ} / 70$ & 4.83 & $\begin{array}{l}\text { BJ negligible nitrate } \\
\text { content }\end{array}$ & 1 & 120 & $\begin{array}{l}\text { 8-s bouts of high-intensity } \\
\text { intermittent-sprint test }\end{array}$ & Work (kJ) & $\begin{array}{l}S=49.2(\text { SD } 24.2) \\
N S=57.8(\text { SD } 34.0) \\
D=\text { yes }\end{array}$ \\
\hline$\underset{(3)^{(66)}}{\operatorname{Martin} \text { et al. }}$ & $\begin{array}{c}16\left(90^{+}\right. \\
\text {and } \\
7 \text { o })\end{array}$ & Team-sport players & $47 \cdot 2$ & 8.5 & $\mathrm{BJ} / 70$ & 4.83 & $\begin{array}{l}\text { BJ negligible nitrate } \\
\text { content }\end{array}$ & 1 & 120 & $\begin{array}{l}\text { 8-second bouts of high- } \\
\text { intensity intermittent- } \\
\text { sprint test }\end{array}$ & $\begin{array}{l}\text { Mean power } \\
\text { output (W) }\end{array}$ & $\begin{array}{l}S=447(\text { SD } 104) \\
N S=444(\text { SD } 117) \\
D=\text { no }\end{array}$ \\
\hline $\begin{array}{l}\text { McQuillan } \\
\text { et al. (1) }\end{array}$ & $9\left(0^{3}\right)^{+1}$ & Cyclists & 68 & 3 & $\mathrm{BJ} / 140$ & 8.0 & $\begin{array}{l}\text { BJ negligible nitrate } \\
\text { content }\end{array}$ & 4 & 150 & Time trial $1 \mathrm{~km}$ & Time (s) & $\begin{array}{l}S=79 \cdot 6(\mathrm{sD} 3 \cdot 5) \\
N S=79 \cdot 2(\mathrm{sD} 2 \cdot 9) \\
D=\text { no }\end{array}$ \\
\hline $\begin{array}{l}\text { McQuillan } \\
\text { et al. (2) }\end{array}$ & $9\left({ }^{*}\right)$ & Cyclists & 68 & 3 & $\mathrm{BJ} / 140$ & 8.0 & $\begin{array}{l}\text { BJ negligible nitrate } \\
\text { content }\end{array}$ & 4 & 150 & Time trial $1 \mathrm{~km}$ & $\begin{array}{l}\text { Mean power } \\
\text { output (W) }\end{array}$ & $\begin{array}{l}S=495(\mathrm{sD} 61 \\
\mathrm{NS}=503(\mathrm{SD} 51) \\
\mathrm{D}=\mathrm{no}\end{array}$ \\
\hline
\end{tabular}




\section{N's British Journal of Nutrition}

\begin{tabular}{|c|c|c|c|c|c|c|c|c|c|c|c|c|}
\hline \multirow[b]{3}{*}{ References } & \multirow{3}{*}{$\begin{array}{l}\text { No. of } \\
\text { subjects } \\
\left(\delta^{\prime},+\right)\end{array}$} & \multirow[b]{3}{*}{ Characteristics of subjects } & \multicolumn{7}{|c|}{ Nitrate supplementation } & \multirow[b]{3}{*}{ Exercise protocol } & \multirow{3}{*}{$\begin{array}{l}\text { Measure of } \\
\text { physical } \\
\text { performance }\end{array}$} & \multirow[b]{3}{*}{ Results } \\
\hline & & & \multicolumn{2}{|c|}{$\begin{array}{c}\mathrm{VO}_{2 \text { peak }} / \\
\mathrm{VO}_{2 \max }(\mathrm{ml} / \mathrm{kg} \\
\text { per min }) \\
\end{array}$} & \multirow{2}{*}{$\begin{array}{l}\text { Ingested fluid/ } \\
\text { volume (ml) }\end{array}$} & \multirow[b]{2}{*}{ Dose (mmol) } & \multirow[b]{2}{*}{ Placebo substance } & \multirow[b]{2}{*}{$\begin{array}{l}\text { Days of } \\
\text { supplementation }\end{array}$} & \multirow[b]{2}{*}{$\begin{array}{c}\text { Time before } \\
\text { trial }(\mathrm{min})\end{array}$} & & & \\
\hline & & & Mean & SD & & & & & & & & \\
\hline $\begin{array}{l}\text { McQuillan } \\
\text { et al. (3) }\end{array}$ & $9(0)$ & Cyclists & 68 & 3 & $\mathrm{BJ} / 140$ & 8.0 & $\begin{array}{l}\text { BJ negligible nitrate } \\
\text { content }\end{array}$ & 7 & 150 & Time trial $1 \mathrm{~km}$ & Time (s) & $\begin{array}{l}S=79 \cdot 3(\mathrm{sD} 3 \cdot 3) \\
N S=79 \cdot 0(\mathrm{sD} 3 \cdot 0) \\
D=\text { no }\end{array}$ \\
\hline $\begin{array}{l}\text { McQuillan } \\
\text { et al. (4) }\end{array}$ & $9\left(0^{*}\right)$ & Cyclists & 68 & 3 & $\mathrm{BJ} / 140$ & 8.0 & $\begin{array}{l}\text { BJ negligible nitrate } \\
\text { content }\end{array}$ & 7 & 150 & Time trial $1 \mathrm{~km}$ & $\begin{array}{l}\text { Mean power } \\
\text { output (W) }\end{array}$ & $\begin{array}{l}S=501(\text { sD 59) } \\
N S=505(\text { SD 52) } \\
D=\text { no }\end{array}$ \\
\hline $\begin{array}{l}\text { McQuillan } \\
\text { et al. (5) }\end{array}$ & $9($ () & Cyclists & 68 & 3 & $\mathrm{BJ} / 140$ & 8.0 & $\begin{array}{l}\text { BJ negligible nitrate } \\
\text { content }\end{array}$ & 3 & 150 & Time trial $4 \mathrm{~km}$ & Time (s) & $\begin{array}{l}S=341(S D 12) \\
N S=340(S D 10) \\
D=\text { no }\end{array}$ \\
\hline $\begin{array}{l}\text { McQuillan } \\
\text { et al. }(6)^{(67)}\end{array}$ & $9\left(0^{7}\right)$ & Cyclists & 68 & 3 & $\mathrm{BJ} / 140$ & 8.0 & $\begin{array}{l}\text { BJ negligible nitrate } \\
\text { content }\end{array}$ & 3 & 150 & Time trial $4 \mathrm{~km}$ & $\begin{array}{l}\text { Mean power } \\
\text { output (W) }\end{array}$ & $\begin{array}{l}S=390(\mathrm{SD} 45) \\
\mathrm{NS}=393(\mathrm{SD} 37)\end{array}$ \\
\hline $\begin{array}{l}\text { McQuillan } \\
\quad \text { et al. }(7)^{(67)}\end{array}$ & $9\left(0^{7}\right)$ & Cyclists & 68 & 3 & $\mathrm{BJ} / 140$ & 8.0 & $\begin{array}{l}\text { BJ negligible nitrate } \\
\text { content }\end{array}$ & 6 & 150 & Time trial $4 \mathrm{~km}$ & Time (s) & $\begin{array}{l}D=\text { no } \\
S=340(\text { SD } 10) \\
N S=340(\text { SD } 11) \\
D=\text { no }\end{array}$ \\
\hline $\begin{array}{l}\text { McQuillan } \\
\text { et al. (8) }\end{array}$ & $9\left(0^{*}\right)$ & Cyclists & 68 & 3 & $\mathrm{BJ} / 140$ & 8.0 & $\begin{array}{l}\text { BJ negligible nitrate } \\
\text { content }\end{array}$ & 6 & 150 & Time trial $4 \mathrm{~km}$ & $\begin{array}{l}\text { Mean power } \\
\text { output (W) }\end{array}$ & $\begin{array}{l}S=394(\mathrm{SD} 38) \\
N S=393(\mathrm{sD} 37) \\
\mathrm{D}=\text { no }\end{array}$ \\
\hline $\begin{array}{l}\text { McQuillan } \\
\text { et al. (1) }\end{array}$ & $8\left(0^{7}\right)$ & Cyclists & 63 & 4 & $\mathrm{BJ} / 70$ & 4.0 & $\begin{array}{l}\text { BJ negligible nitrate } \\
\text { content }\end{array}$ & 8 & 120 & Time trial $4 \mathrm{~km}$ & Time (s) & $\begin{array}{l}S=343 \cdot 6(\mathrm{SD} 14 \cdot 3) \\
\mathrm{NS}=344 \cdot 8(\mathrm{SD} \\
14 \cdot 0)\end{array}$ \\
\hline $\begin{array}{l}\text { McQuillan } \\
\quad \text { et al. }(2)^{(68)}\end{array}$ & $8\left({ }^{7}\right)$ & Cyclists & 63 & 4 & $\mathrm{BJ} / 70$ & 4.0 & $\begin{array}{l}\text { BJ negligible nitrate } \\
\text { content }\end{array}$ & 8 & 120 & Time trial $4 \mathrm{~km}$ & $\begin{array}{l}\text { Mean power } \\
\text { output }\end{array}$ & $\begin{array}{l}D=n o \\
S=380(\text { sD } 41) \\
N S=375(\text { sD } 40) \\
D=n o\end{array}$ \\
\hline $\begin{array}{l}\text { Muggeridge } \\
\text { et al. }(1)^{(69)}\end{array}$ & $8\left({ }^{7}\right)$ & Kayakers & 49.0 & $6 \cdot 1$ & $\mathrm{BJ} / 70$ & $5 \cdot 0$ & Tomato juice & 1 & 180 & $\begin{array}{l}\text { Steady-state paddling at } \\
60 \% \text { of } W R_{\max }(15 \mathrm{~min})\end{array}$ & $\begin{array}{l}\text { Mean power } \\
\text { output (W) }\end{array}$ & $\begin{array}{l}S=108(\mathrm{sD} 64 \cdot 8) \\
N S=108(\mathrm{sD} 62 \cdot 0) \\
D=\text { no }\end{array}$ \\
\hline $\begin{array}{l}\text { Muggeridge } \\
\text { et al. (2) }\end{array}$ & $8\left({ }^{*}\right)$ & Kayakers & 49.0 & $6 \cdot 1$ & $\mathrm{BJ} / 70$ & 5.0 & Tomato juice & 1 & 180 & Time trial $1 \mathrm{~km}$ & Time (s) & $\begin{array}{l}S=276(\text { SD 14.1) } \\
N S=277(\text { SD 14.1) } \\
D=\text { no }\end{array}$ \\
\hline $\begin{array}{l}\text { Nyakayiru } \\
\text { et al. }^{(70)}\end{array}$ & $17\left(\delta^{\lambda}\right)$ & Cyclists and triathletes & $65 \cdot 0$ & 4.0 & Sodium nitrate/140 & $12 \cdot 9$ & Sodium chloride & 6 & 240 & Time trial $10 \mathrm{~km}$ & Time (s) & $\begin{array}{l}S=1004(\mathrm{SD} 67) \\
N S=1017(\mathrm{SD} 71) \\
D=\text { no }\end{array}$ \\
\hline $\begin{array}{l}\text { Peacock } \\
\text { et al. }{ }^{(28)}\end{array}$ & $10\left(\emptyset^{\lambda}\right)$ & Elite cross-country skiers & $69 \cdot 6$ & $5 \cdot 1$ & $\begin{array}{l}1 \mathrm{~g} \text { of potassium } \\
\text { nitrate in a capsule }\end{array}$ & 9.9 & $\begin{array}{l}1 \mathrm{~g} \text { of maltodextrin in a } \\
\text { capsule }\end{array}$ & 1 & 150 & Time trial $5 \mathrm{~km}$ & Time (s) & $\begin{array}{l}S=1005(\text { sD 53) } \\
N S=996(S D 49) \\
D=\text { no }\end{array}$ \\
\hline $\begin{array}{l}\text { Peeling et al. } \\
(1)^{(71)}\end{array}$ & $6\left(\delta^{*}\right)$ & Kayakers & 57.15 & $2 \cdot 77$ & $\mathrm{BJ} / 70$ & $4 \cdot 8$ & $\begin{array}{l}\text { BJ negligible nitrate } \\
\text { content }\end{array}$ & 1 & 150 & $\begin{array}{l}\text { 4-min all-out maximal effort } \\
\text { on the stationary kayak } \\
\text { ergometer }\end{array}$ & $\begin{array}{l}\text { Power output } \\
\text { (W) }\end{array}$ & $\begin{array}{l}S=319(\text { SD } 35) \\
N S=318(\text { SD } 42) \\
D=\text { no }\end{array}$ \\
\hline $\begin{array}{l}\text { Peeling et al. } \\
\text { (2) }\end{array}$ & $6\left({ }^{\top}\right)$ & Kayakers & $57 \cdot 15$ & $2 \cdot 77$ & $\mathrm{BJ} / 70$ & $4 \cdot 8$ & $\begin{array}{l}\text { BJ negligible nitrate } \\
\text { content }\end{array}$ & 1 & 150 & $\begin{array}{l}\text { 4-min all-out maximal } \\
\text { effort on the stationary } \\
\text { kayak ergometer }\end{array}$ & $\begin{array}{l}\text { Distance } \\
\quad \text { covered }(m)\end{array}$ & $\begin{array}{l}S=989(\mathrm{sD} 31) \\
N S=982(\operatorname{sD~36}) \\
D=\text { no }\end{array}$ \\
\hline $\begin{array}{l}\text { Peeling et al. } \\
(3)^{(71)}\end{array}$ & 5 (\%) & Kayakers & $47 \cdot 8$ & 3.7 & $\mathrm{BJ} / 70$ & $9 \cdot 6$ & $\begin{array}{l}\text { BJ negligible nitrate } \\
\text { content }\end{array}$ & 1 & 120 & Time trial $500 \mathrm{~m}$ & Time (s) & $\begin{array}{l}S=114 \cdot 6(\mathrm{sD} 1 \cdot 5) \\
N S=116 \cdot 7(\mathrm{SD} 2 \cdot 2) \\
D=\text { yes }\end{array}$ \\
\hline $\begin{array}{l}\text { Peeling et al. } \\
\qquad(4)^{(71)}\end{array}$ & 5 (官) & Kayakers & $47 \cdot 8$ & 3.7 & $\mathrm{BJ} / 70$ & 9.6 & $\begin{array}{l}\text { BJ negligible nitrate } \\
\text { content }\end{array}$ & 1 & 120 & Time trial $500 \mathrm{~m}$ & $\begin{array}{l}\text { Velocity in 100- } \\
400 \mathrm{~m}(\mathrm{~m} / \mathrm{s})\end{array}$ & $\begin{array}{l}\mathrm{S}=4.4(\mathrm{SD} 0.03) \\
\mathrm{NS}=4.3(\mathrm{SD} 0.05) \\
\mathrm{D}=\text { yes }\end{array}$ \\
\hline$\underset{(1)^{(72)}}{\operatorname{Rimer} \text { et al. }}$ & $\begin{array}{c}13(11 \text { o } \\
\text { and } \\
2 \text { of })\end{array}$ & $\begin{array}{l}\text { Tennis, Alpine Ski, American } \\
\text { Football, Cycling, Triathlon }\end{array}$ & & - & $\mathrm{BJ} / 140(70+70)$ & $11 \cdot 2$ & $\begin{array}{l}\text { BJ negligible nitrate } \\
\text { content }\end{array}$ & 1 & 150 & $\begin{array}{l}4 \times, \text { maximal inertial-load } \\
\text { cycling trial } \\
(3-4 \mathrm{~s})\end{array}$ & $\begin{array}{l}\text { Maximal power } \\
\text { output (W) }\end{array}$ & $\begin{array}{l}S=1229(\mathrm{sD} 317) \\
N S=1213(\mathrm{SD} 300) \\
D=\text { yes }\end{array}$ \\
\hline $\begin{array}{l}\text { Rimer et al. } \\
(2)^{(72)}\end{array}$ & $\begin{array}{c}13\left(11 \sigma^{+}\right. \\
\text {and } \\
20)\end{array}$ & $\begin{array}{l}\text { Tennis, Alpine Ski, American } \\
\text { Football, Cycling, Triathlon }\end{array}$ & & - & $\mathrm{BJ} / 140(70+70)$ & $11 \cdot 2$ & $\begin{array}{l}\text { BJ negligible nitrate } \\
\text { content }\end{array}$ & 1 & 150 & $\begin{array}{l}\text { Maximal isokinetic cycling } \\
\text { trial, } 120 \mathrm{rpm}(30 \mathrm{~s})\end{array}$ & Total work (kJ) & $\begin{array}{l}S=22 \cdot 8(\mathrm{SD} 4 \cdot 8) \\
\mathrm{NS}=23 \cdot 0(4 \cdot 4) \\
\mathrm{D}=\mathrm{n}\end{array}$ \\
\hline Rimer et al. ${ }^{(73)}$ & $\begin{array}{l}13(110 \\
\text { and } \\
2 \text { o })\end{array}$ & $\begin{array}{l}\text { Tennis, Alpine Ski, American } \\
\text { Football, Cycling, Triathlon }\end{array}$ & & - & $\mathrm{BJ} / 140(70+70)$ & $11 \cdot 2$ & $\begin{array}{l}\text { BJ negligible nitrate } \\
\text { content }\end{array}$ & 1 & 150 & $\begin{array}{l}\text { Maximal isokinetic } \\
\text { cycling trial, } \\
120 \mathrm{rpm}(30 \mathrm{~s})\end{array}$ & Peak Power (W) & $\begin{array}{l}S=1173(\text { sD 255) } \\
N S=1185(\text { SD 249) } \\
D=\text { no }\end{array}$ \\
\hline
\end{tabular}




\section{N British Journal of Nutrition}

Table 2. Continued

\begin{tabular}{|c|c|c|c|c|c|c|c|c|c|c|c|c|}
\hline \multirow[b]{3}{*}{ References } & \multirow{3}{*}{$\begin{array}{l}\begin{array}{l}\text { No. of } \\
\text { subjects } \\
\left(\delta^{\star}, \text { O }\right)\end{array} \\
\end{array}$} & \multirow[b]{3}{*}{ Characteristics of subjects } & \multicolumn{7}{|c|}{ Nitrate supplementation } & \multirow[b]{3}{*}{ Exercise protocol } & \multirow{3}{*}{$\begin{array}{l}\text { Measure of } \\
\text { physical } \\
\text { performance }\end{array}$} & \multirow[b]{3}{*}{ Results } \\
\hline & & & \multicolumn{2}{|c|}{$\begin{array}{c}\mathrm{VO}_{2 \text { peak }} / \\
\mathrm{VO}_{2 \max }(\mathrm{ml} / / \mathrm{kg} \\
\text { per min) } \\
\end{array}$} & \multirow{2}{*}{$\begin{array}{l}\text { Ingested fluid/ } \\
\text { volume (ml) }\end{array}$} & \multirow[b]{2}{*}{ Dose $(\mathrm{mmol})$} & \multirow[b]{2}{*}{ Placebo substance } & \multirow{2}{*}{$\begin{array}{c}\text { Days of } \\
\text { supplementation }\end{array}$} & \multirow{2}{*}{$\begin{array}{c}\text { Time before } \\
\text { trial (min) }\end{array}$} & & & \\
\hline & & & Mean & SD & & & & & & & & \\
\hline $\begin{array}{l}\text { Shannon et al. } \\
(1)^{(74)}\end{array}$ & $8\left(0^{*}\right)$ & Runners or triathletes & $62 \cdot 3$ & $8 \cdot 1$ & $\mathrm{BJ} / 140$ & 12.5 & $\begin{array}{l}\text { BJ negligible nitrate } \\
\text { content }\end{array}$ & 1 & 180 & Time trial $1.500 \mathrm{~m}$ & Time (s) & $\begin{array}{c}\mathrm{S}=319 \cdot 6(\mathrm{sD} 36 \cdot 2) \\
\mathrm{NS}=325 \cdot 7(\mathrm{SD} \\
38 \cdot 8)\end{array}$ \\
\hline $\begin{array}{l}\text { Shannon et al. } \\
(2)^{(74)}\end{array}$ & $8\left({ }^{3}\right)$ & Runners or triathletes & $62 \cdot 3$ & $8 \cdot 1$ & $\mathrm{BJ} / 140$ & 12.5 & $\begin{array}{l}\text { BJ negligible nitrate } \\
\text { content }\end{array}$ & 1 & 180 & Time trial $10.000 \mathrm{~m}$ & Time (s) & $\begin{array}{c}\mathrm{D}=\text { yes } \\
\mathrm{S}=2643 \cdot 1(\mathrm{sD} \\
324 \cdot 1) \\
\mathrm{NS}=2649 \cdot 9(\mathrm{sD} \\
319 \cdot 8) \\
\mathrm{D}=\mathrm{no}\end{array}$ \\
\hline $\begin{array}{l}\text { Thompson } \\
\text { et al. (1) }\end{array}$ & $36\left(0^{\pi}\right)$ & Team-sport players & & - & $\mathrm{BJ} / 70$ & $6 \cdot 4$ & $\begin{array}{l}\text { BJ negligible nitrate } \\
\text { content }\end{array}$ & 5 & 150 & Sprints $(5 \times 20 \mathrm{~m})$ & Time (s) at $20 \mathrm{~m}$ & $\begin{array}{l}S=3.98(\text { sD } 0.18) \\
N S=4.03(\text { (sD } 0.19) \\
D=\text { ves }\end{array}$ \\
\hline $\begin{array}{l}\text { Thompson } \\
\text { et al. (2) }\end{array}$ & $36\left(0^{7}\right)$ & Team-sport players & & - & $\mathrm{BJ} / 70$ & $6 \cdot 4$ & $\begin{array}{l}\text { BJ negligible nitrate } \\
\text { content }\end{array}$ & 5 & 150 & $\begin{array}{l}\text { Teste Yo-Yo IR1 } \\
(2 \times 20 \mathrm{~m})\end{array}$ & $\begin{array}{l}\text { Distance } \\
\text { covered }(m)\end{array}$ & $\begin{array}{l}S=1422(\mathrm{SD} 502) \\
\mathrm{NS}=1369(\mathrm{SD} 505)\end{array}$ \\
\hline $\begin{array}{l}\text { Wilkerson } \\
\text { et al. (1) }\end{array}$ & $8\left({ }^{7}\right)$ & Cyclists & 63 & 8 & $\mathrm{BJ} / 500$ & $6 \cdot 2$ & $\begin{array}{l}\text { BJ negligible nitrate } \\
\text { content }\end{array}$ & 1 & 150 & Time trial 50 miles & Time (min) & $\begin{array}{l}S=136 \cdot 7(\text { sD } 5 \cdot 6) \\
N S=137.9(\text { sD } 6.4) \\
D=n O\end{array}$ \\
\hline $\begin{array}{l}\text { Wilkerson } \\
\quad \text { et al. (2) }\end{array}$ & $8\left({ }^{\pi}\right)$ & Cyclists & 63 & 8 & $\mathrm{BJ} / 500$ & $6 \cdot 2$ & $\begin{array}{l}\text { BJ negligible nitrate } \\
\text { content }\end{array}$ & 1 & 150 & Time trial 50 miles & $\begin{array}{l}\text { Mean power } \\
\text { output (W) }\end{array}$ & $\begin{array}{l}\mathrm{S}=238(\mathrm{sD} 22) \\
\mathrm{NS}=235(\mathrm{sD} 27) \\
\mathrm{D}=\mathrm{no}\end{array}$ \\
\hline
\end{tabular}

o, Male; , female; BJ, beetroot juice; NR, not reported; S, supplemented; NS, no supplementation; D, statistical difference. 


\begin{tabular}{|c|c|c|c|}
\hline Study & SMD & $95 \% \mathrm{Cl}$ & Weight $(\%)$ \\
\hline Christensen et al. (1) ${ }^{(42)}$ & -0.15 & $-1 \cdot 13,0 \cdot 84$ & $1 \cdot 82$ \\
\hline Vanhatalo et al. ${ }^{(16)}$ & -0.12 & $-1 \cdot 10,0 \cdot 86$ & $1 \cdot 82$ \\
\hline Fulford et al. $(1)^{(45)}$ & -0.12 & $-1 \cdot 10,0 \cdot 86$ & $1 \cdot 82$ \\
\hline Coggan et al. $(1)^{(43)}$ & -0.11 & $-0.91,0.69$ & $2 \cdot 73$ \\
\hline Coggan et al. (2) & -0.08 & $-0.88,0.72$ & $2 \cdot 73$ \\
\hline Fulford et al. (2) ${ }^{(45)}$ & -0.07 & $-1.05,0.91$ & 1.82 \\
\hline Wylie et al. (2) & -0.04 & $-0.92,0.83$ & $2 \cdot 28$ \\
\hline Buck et al. ${ }^{(41)}$ & -0.03 & $-0.53,0.48$ & 6.84 \\
\hline Wylie et al. (3) ${ }^{(57)}$ & -0.02 & $-0.89,0.86$ & $2 \cdot 28$ \\
\hline Vasconcellos et al. (55) & -0.01 & $-0 \cdot 79,0 \cdot 78$ & 2.85 \\
\hline Bailey et al. (1) & 0.03 & $-1 \cdot 01,1 \cdot 08$ & $1 \cdot 60$ \\
\hline Murphy et al. ${ }^{\left({ }^{49)}\right.}$ & 0.05 & $-0.79,0.88$ & $2 \cdot 51$ \\
\hline Coggan et al. (3) & 0.05 & $-0.75,0.85$ & $2 \cdot 74$ \\
\hline Porcelli et al. $(3)^{(51)}$ & 0.07 & $-1 \cdot 06,1 \cdot 20$ & $1 \cdot 37$ \\
\hline Rienks et al. & 0.09 & $-0.84,1.01$ & $2 \cdot 05$ \\
\hline Porcelli et al. (2) & 0.12 & $-0.93,1.17$ & 1.59 \\
\hline Coggan et al. (4) & 0.16 & $-0.64,0.97$ & $2 \cdot 73$ \\
\hline Wylie et al. (1) $)^{(57)}$ & 0.21 & $-0.67,1.09$ & $2 \cdot 27$ \\
\hline Thompson et al. ${ }^{(53)}$ & 0.22 & $-0.48,0.91$ & 3.63 \\
\hline Thompson et al. ${ }^{(54)}$ & 0.22 & $-0.47,0.92$ & 3.63 \\
\hline Wylie et al. ${ }^{(56)}$ & 0.23 & $-0.51,0.97$ & $3 \cdot 17$ \\
\hline Nyakayiru et al. ${ }^{(50)}$ & 0.26 & $-0.44,0.95$ & $3 \cdot 62$ \\
\hline Christensen et al. (2) ${ }^{(42)}$ & $0 \cdot 30$ & $-0 \cdot 69,1 \cdot 28$ & $1 \cdot 80$ \\
\hline Kokkinoplitis and chester ${ }^{(47)}$ & 0.31 & $-0.75,1 \cdot 36$ & 1.58 \\
\hline Porcelli et al. (1) & 0.31 & $-0 \cdot 68,1 \cdot 29$ & 1.80 \\
\hline Fulford et al. (3) $)^{(45)}$ & 0.38 & $-0.61,1.37$ & 1.79 \\
\hline Wylie et al. (1) ${ }^{(17)}$ & 0.41 & $-0.47,1.30$ & $2 \cdot 23$ \\
\hline Corry et al. ${ }^{(44)}$ & 0.43 & $-0.46,1 \cdot 31$ & $2 \cdot 22$ \\
\hline Larsen et al. ${ }^{(15)}$ & 0.43 & $-0.51,1.36$ & $2 \cdot 00$ \\
\hline Aucouturier et al. (2) ${ }^{(38)}$ & 0.45 & $-0.36,1 \cdot 26$ & $2 \cdot 66$ \\
\hline Aucouturier et al. (1) (38) & 0.50 & $-0.32,1 \cdot 31$ & $2 \cdot 65$ \\
\hline Kelly et al. (4) & 0.50 & $-0 \cdot 44,1 \cdot 44$ & 1.98 \\
\hline Wylie et al. (3) $)^{(17)}$ & 0.51 & $-0.38,1.40$ & $2 \cdot 20$ \\
\hline Mosher et al. ${ }^{(48)}$ & 0.52 & $-0 \cdot 30,1 \cdot 33$ & $2 \cdot 64$ \\
\hline Bailey et al. ${ }^{(18)}$ & 0.52 & $-0 \cdot 48,1 \cdot 52$ & 1.76 \\
\hline Breese et al. ${ }^{(40)}$ & 0.53 & $-0.41,1.48$ & 1.97 \\
\hline Wylie et al. (2) ${ }^{(17)}$ & 0.54 & $-0.36,1 \cdot 43$ & $2 \cdot 19$ \\
\hline Bailey et al. (2) (39) & 0.58 & $-0.49,1.65$ & 1.52 \\
\hline Bailey et al. ${ }^{(19)}$ & 0.59 & $-0.49,1.66$ & 1.52 \\
\hline Kelly et al. (3) ${ }^{(46)}$ & 0.62 & $-0.33,1.57$ & 1.95 \\
\hline Kelly et al. (2) ${ }^{(46)}$ & 0.64 & $-0.31,1.59$ & 1.94 \\
\hline Lansley et al. ${ }^{(23)}$ & 0.66 & $-0.29,1.62$ & 1.93 \\
\hline Kelly et al. (1) $(46)$ & 1.06 & $0.06,2.05$ & $1 \cdot 77$ \\
\hline Overall $\left(I^{2}=0.0 \%, P=1.000\right)$ & 0.25 & $0 \cdot 12,0.38$ & $100 \cdot 00$ \\
\hline 1 & 1 & & \\
\hline
\end{tabular}

Fig. 3. Forest plot of physical performance following dietary $\mathrm{NO}_{3}^{-}$supplementation in non-athletes. SMD, standardised mean difference.

analysis, heterogeneity was observed among these studies $\left(I^{2}=0 \% ; Q=13 \cdot 31, \mathrm{df}=16, P=0 \cdot 65\right)$.

Non-athletes subjected to long-duration tests. After pooling the data from twenty-five trials, the mean effect size was 0.33 (95\% CI $0 \cdot 15,0 \cdot 51$ ), which indicates that the dietary $\mathrm{NO}_{3}^{-}$supplementation had a small and significant beneficial effect on physical performance $(P<0 \cdot 05$; Fig. 7$)$. According to a fixedeffects analysis, no heterogeneity was observed among these studies $\left(I^{2}=0 \% ; Q=8 \cdot 01, \mathrm{df}=24, P=0.99\right)$.

Athletes subjected to long-duration tests. After pooling the data from forty-four trials, the mean effect size was 0.05 (95\% CI $-0.07,0 \cdot 17$ ), which indicates that the dietary $\mathrm{NO}_{3}^{-}$supplementation had a negligible and non-significant effect on physical performance ( $P>0.05$; Fig. 8$)$. According to a fixed-effects analysis, no heterogeneity was observed among these studies $\left(I^{2}=0 \% ; Q=4.82, \mathrm{df}=43, P=1 \cdot 00\right)$. The subsequent analysis consisted of subdividing the non-athletes that performed longduration tests according to the test protocol used.
Non-athletes subjected to long-duration, open-ended tests. After pooling the data from fourteen trials, the mean effect size was 0.47 (95\% CI $0.23,0.71)$, which indicates that the dietary $\mathrm{NO}_{3}^{-}$supplementation had a small and significant beneficial effect on physical performance $(P<0.05$; Fig. 9). According to a fixed-effects analysis, no heterogeneity was observed among these studies $\left(I^{2}=0 \% ; Q=3 \cdot 77, \mathrm{df}=13\right.$, $P=0.99)$.

Non-athletes subjected to long-duration time trials. After pooling the data from four trials, the mean effect size was $0 \cdot 12$ (95\% CI $-0.37,0.61$ ), which indicates that the dietary $\mathrm{NO}_{3}^{-}$ supplementation had a negligible and non-significant effect on physical performance ( $P>0.05$; Fig. 10). According to a fixedeffects analysis, no heterogeneity was observed among these studies $\left(I^{2}=0 \% ; Q=0 \cdot 16, \mathrm{df}=3, P=0 \cdot 98\right)$.

Non-athletes subjected to long-duration, graded-exercise tests. After pooling the data from five trials, the mean effect size was $0.20(95 \% \mathrm{CI}-0.18,0.59)$, which indicates that the dietary $\mathrm{NO}_{3}^{-}$supplementation had a small but non-significant effect on 


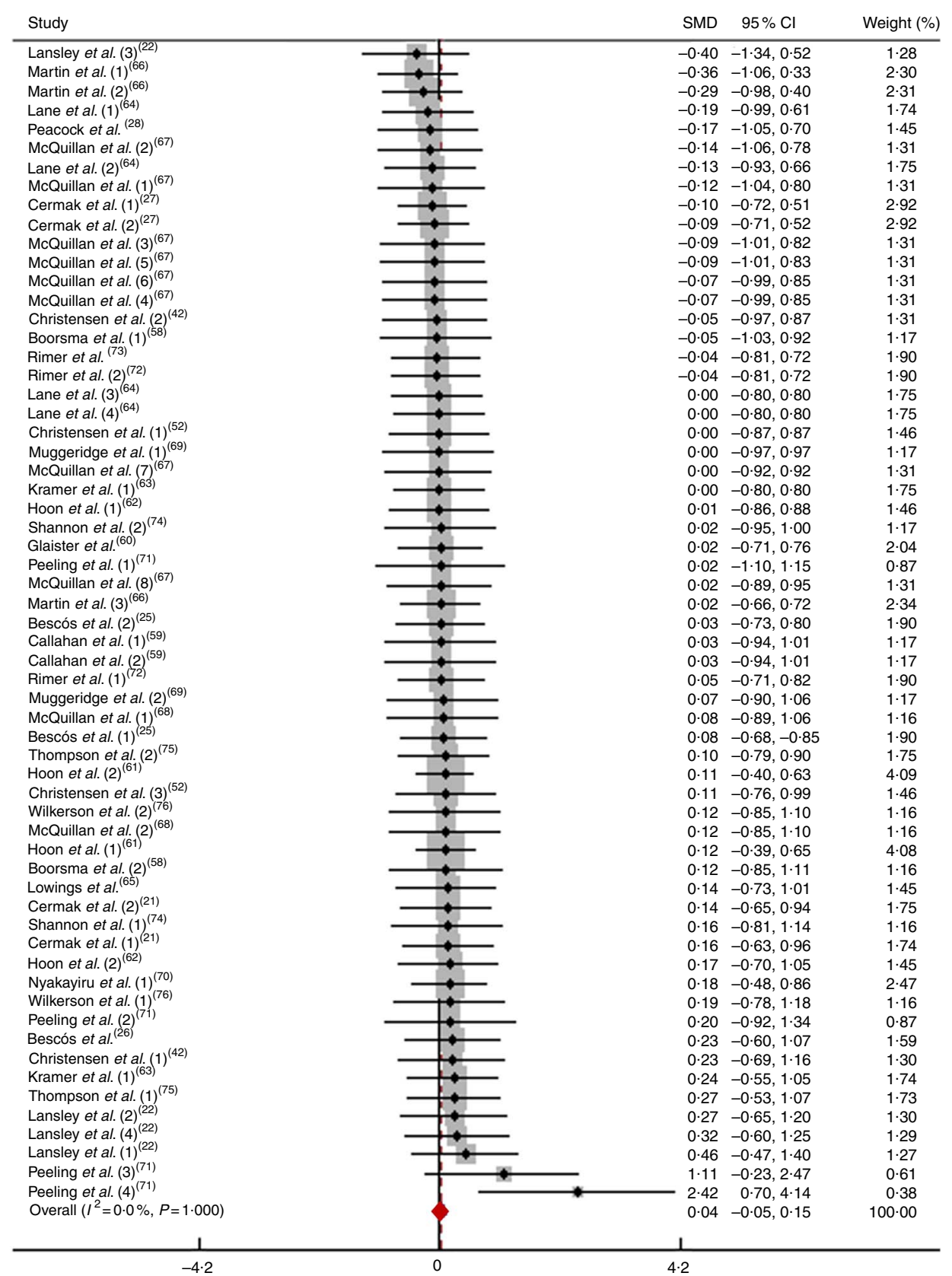

Fig. 4. Forest plot of physical performance following dietary $\mathrm{NO}_{3}^{-}$supplementation in athletes. SMD, standardised mean difference.

physical performance ( $P>0 \cdot 05$; Fig. 11). According to a fixedeffects analysis, no heterogeneity was observed among these studies $\left(I^{2}=0 \% ; Q=1 \cdot 38, \mathrm{df}=4, P=0 \cdot 84\right)$.

Cyclists. Most tested athletes were cyclists; therefore, this subgroup was subjected to a special analysis in which they were evaluated alone without the inclusion of athletes engaged in other sports. After pooling the data from thirty-seven trials, the effect size mean was 0.04 (95\% CI -0.09, 0.17), which indicates that the dietary $\mathrm{NO}_{3}^{-}$supplementation had a negligible and non-significant effect on physical performance ( $P>0.05$; Fig. 12). According to a fixed-effects analysis, heterogeneity was observed among these studies $\left(I^{2}=0 \% ; Q=4 \cdot 90, \mathrm{df}=36, P=1 \cdot 00\right)$.
Analysis of the relationship between the performance level and the ergogenic response to the $\mathrm{NO}_{3}^{-}$supplementation

By analysing the percentage of trials reporting increased performance in individuals classified into different PL, we observed numerous trials, that is, 50 and $56.5 \%$, showing increased performance in individuals with PL1 and PL2, respectively. In contrast, approximately $37 \%$ of the trials involving individuals with PL3 showed an increased performance following the $\mathrm{NO}_{3}^{-}$supplementation, whereas in trials involving individuals with PL4 and PL5 no improvement in performance was observed following the $\mathrm{NO}_{3}^{-}$supplementation (Fig. 13). The $\chi^{2}$ test showed a different distribution among the PL $(P=0.002)$. 


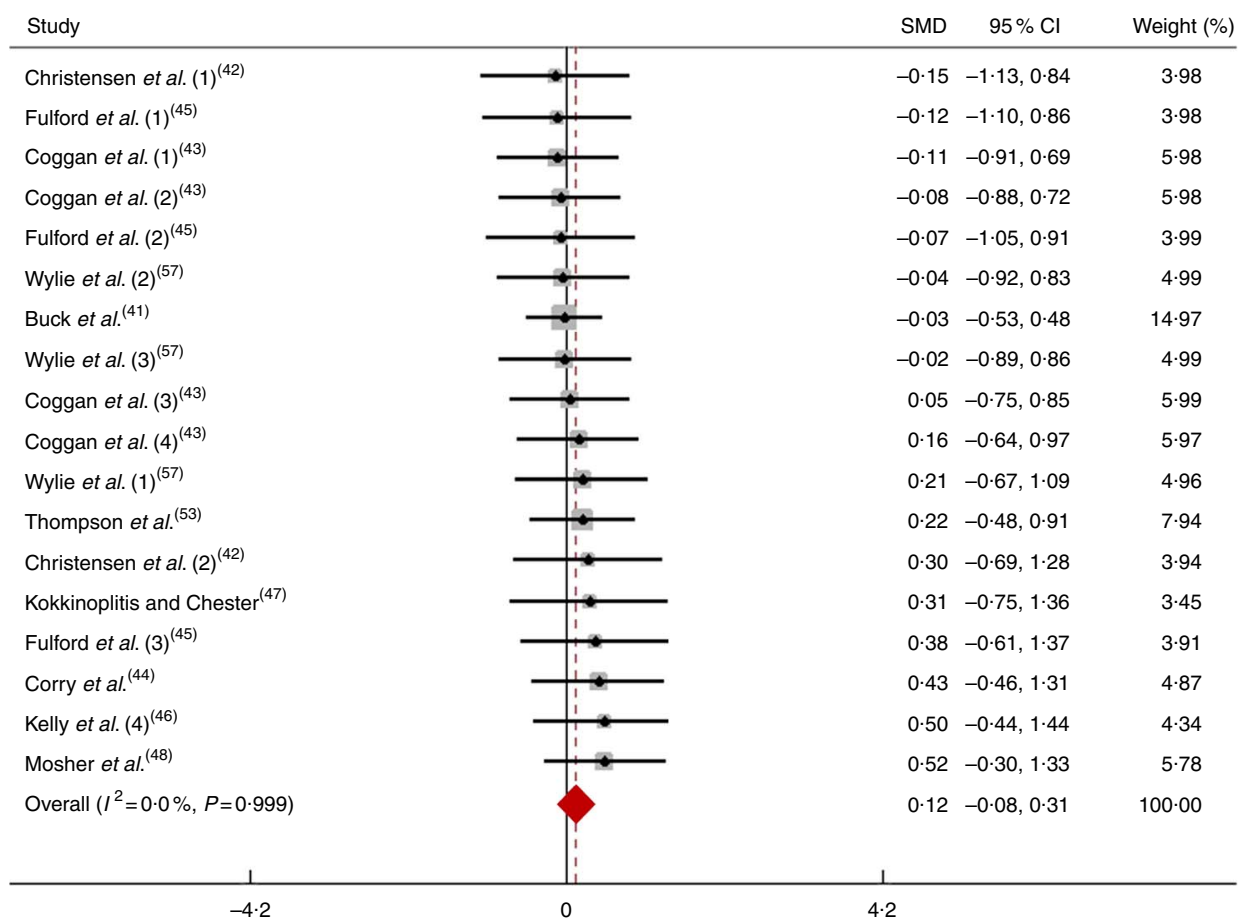

Fig. 5. Forest plot of physical performance during a short-duration test following dietary $\mathrm{NO}_{3}^{-}$supplementation in non-athletes. SMD, standardised mean difference.

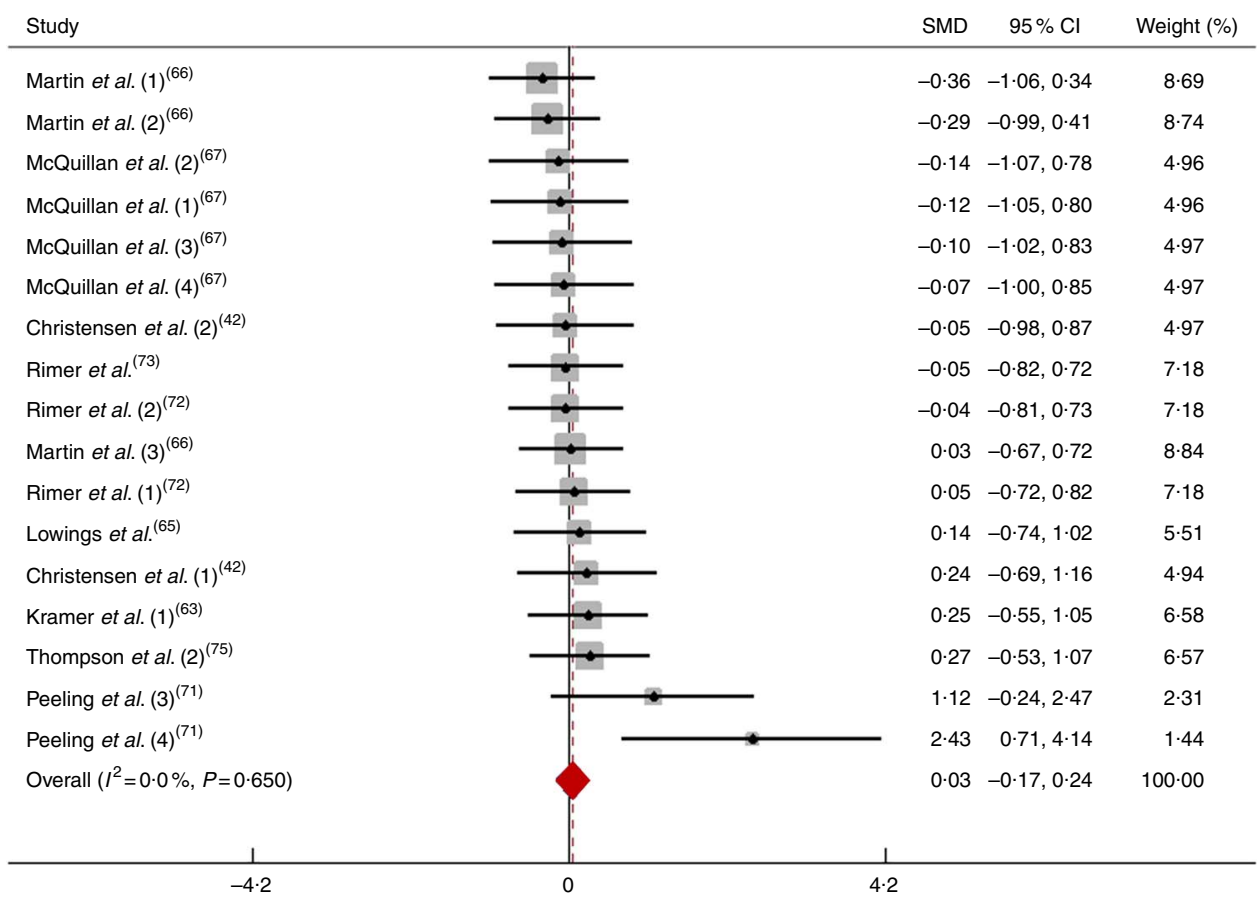

Fig. 6. Forest plot of physical performance during a short-duration test following dietary $\mathrm{NO}_{3}^{-}$supplementation in athletes. SMD, standardised mean difference.

\section{Association between supplementation features and} changes in physical performance

Pearson's correlation analyses were performed to verify the association between these variables, including the association between changes in physical performance and the dose of $\mathrm{NO}_{3}^{-}$(non-athletes: $r 0.351, P>0.05$; athletes: $r 0.099, P>0.05$ ), the number of days of supplementation (non-athletes: $r$ 0.166, $P>0.05$; athletes: $r 0.114, P>0.05$ ) and the total amount ingested (dose multiplied by days under supplementation) (non-athletes: $r$ 0.112, $P>0.05$; athletes: $r 0 \cdot 088, P>0 \cdot 05$ ). No significant correlations were observed between the supplementation features evaluated and changes in physical performance. 


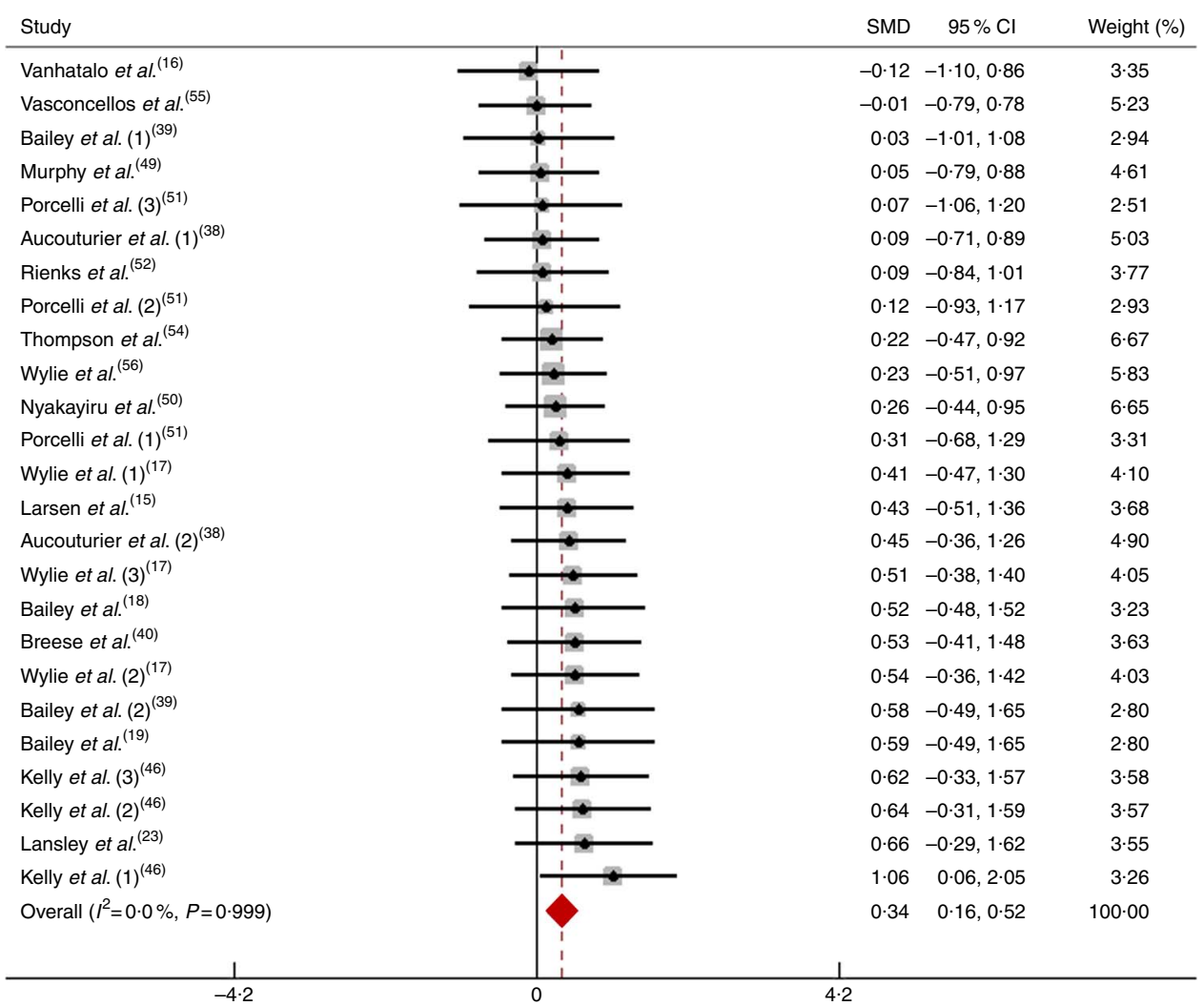

Fig. 7. Forest plot of physical performance during a long-duration test following dietary $\mathrm{NO}_{3}^{-}$supplementation in non-athletes. SMD, standardised mean difference.

\section{Publication bias}

Publication bias was assessed by a visual inspection of the funnel plot for all subgroups analysed: non-athletes (online Supplementary Fig. S2(a)), athletes (online Supplementary Fig. S1(a)), non-athletes subjected to short-duration tests (online Supplementary Fig. S2(b)), athletes subjected to short-duration tests (online Supplementary Fig. S1(b)), non-athletes subjected to long-duration tests (online Supplementary Fig. S2(c)), athletes subjected to long-duration tests (online Supplementary Fig. S1 (c)), non-athletes subjected to long-duration, open-ended tests (online Supplementary Fig. S3(a)), non-athletes subjected to long-duration time trials (online Supplementary Fig. S3(b)) and non-athletes subjected to long-duration, graded-exercise tests (online Supplementary Fig. S3(c)). These analyses revealed minor asymmetrical inverted distributions that were prominent in all plots, suggesting the presence of a small publication bias.

\section{Risk of bias}

The risk of bias was assessed in fifty-four studies (twenty-six and twenty-eight conducted with non-athletes and athletes, respectively) in the systematic review. One study ${ }^{(71)}$ was subjected to two independent evaluations because it presented independent experimental trials. Out of fifty-five evaluations, forty-eight did not present any major risk of bias. Approximately $13 \%$ (non-athletes, two studies; athletes, five studies) of the studies did not blind the participants or researchers. In general, the studies evaluated in the present systematic review showed consistent control of the risk of bias and were deemed to be good-quality studies (online Supplementary Tables S3 and S4).

\section{Discussion}

The present systematic review and meta-analysis demonstrated that the level of physical fitness is a determining factor in the performance-enhancing effects associated with $\mathrm{NO}_{3}^{-}$supplementation. Although athletes are usually less prone to benefit from $\mathrm{NO}_{3}^{-}$supplementation, non-athletes can experience small but significant advantages in their physical performance, particularly in performance evaluations using long-duration, openended tests. Interestingly, this effect is not observed using time trials, which is the most ecologically valid exercise protocol $^{(77)}$. These findings regarding the beneficial effects induced by $\mathrm{NO}_{3}^{-}$ supplementation in non-athletes are supported by the analysis in which the participants were subdivided according to their PL, and those classified at the lower levels (less conditioned) showed more improvements. This information is very important for exercise practitioners and athletes and provides support in decisions regarding whether to use this potential ergogenic aid to improve physical performance and health.

In the present meta-analysis, we observed that individuals with higher fitness levels benefit less from $\mathrm{NO}_{3}^{-}$supplementation (Fig. 13). Consistently, the effect size of $\mathrm{NO}_{3}^{-}$supplementationmediated changes on performance in athletes was mostly irrelevant (Fig. 4). In contrast, non-athletes can benefit from $\mathrm{NO}_{3}^{-}$ supplementation (Fig. 3). This was the first study to systematically show the importance of characterising the fitness levels of 


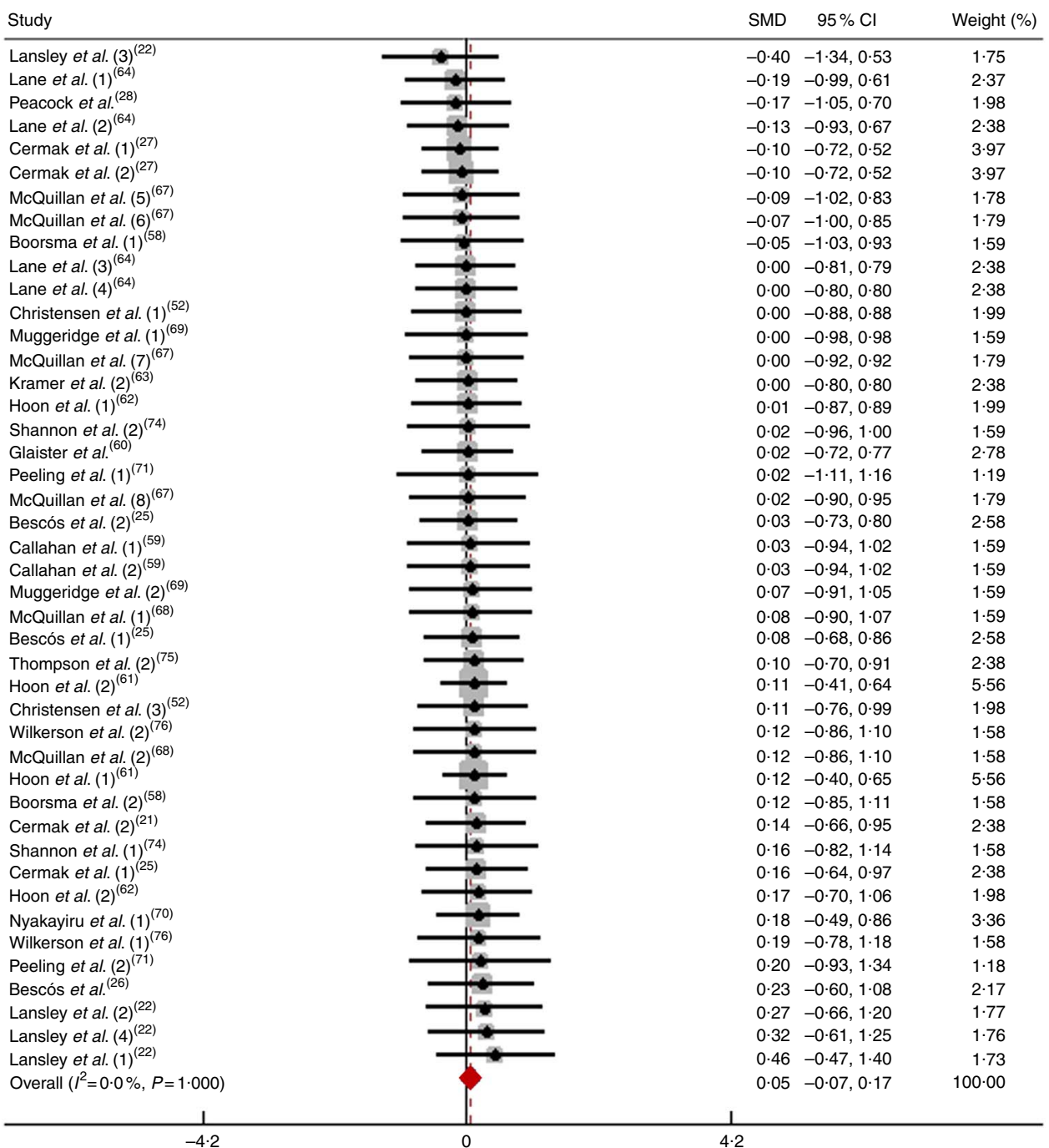

Fig. 8. Forest plot of physical performance during a long-duration test following dietary $\mathrm{NO}_{3}^{-}$supplementation in athletes. SMD, standardised mean difference.

individuals before adopting a nutritional $\mathrm{NO}_{3}^{-}$supplementation ergogenic strategy. Similarly, Porcelli et al. ${ }^{(51)}$ assessed athletic performance in subjects with three aerobic fitness levels after $6 \mathrm{~d}$ of supplementation with $5.5 \mathrm{mmol}$ per $\mathrm{d}$ of $\mathrm{NO}_{3}^{-}$. The authors observed that individuals with lower and moderate aerobic capacities performed better during the time trial after the $\mathrm{NO}_{3}^{-}$ supplementation. However, the performance during the time trial was not improved in individuals with a higher aerobic capacity.

Several mechanisms may act collectively to improve performance following $\mathrm{NO}_{3}^{-}$supplementation in non-athletes, including beneficial effects of an increased NO bioavailability in the skeletal muscles, blood vessels and even in the brain (Fig. 14). In contrast, the mechanisms underlying the limited ergogenic effects of $\mathrm{NO}_{3}^{-}$supplementation in high-performance athletes have not been well elucidated. The ergogenic effects of $\mathrm{NO}_{3}^{-}$supplementation are related to enhanced NO bioavailability, and athletes probably already have optimal levels of $\mathrm{NO}^{(51)}$. Highly trained subjects are likely to have high NOS activity ${ }^{(83)}$, which might render the $\mathrm{NO}_{3}^{-}-\mathrm{NO}_{2}^{-}-\mathrm{NO}$ pathway less important for NO production. Therefore, the resulting increase in NO bioavailability due to supplementation does not appear to be relevant in athletes. In addition to these factors, Porcelli et $a l .{ }^{(51)}$ suggested that high-performance athletes have a high daily energy expenditure and possibly an enriched diet. Therefore, a diet consisting of a higher intake of $\mathrm{NO}_{3}^{-}$in these subjects should be considered. Furthermore, recent evidence that $\mathrm{NO}_{3}^{-}$supplementation may preferentially alter contractile function in type II fibres ${ }^{(79)}$ suggests that endurance athletes, who typically have a low proportion of such fibres in their musculature ${ }^{(84)}$, might experience a blunted physiological response to $\mathrm{NO}_{3}^{-}$supplementation.

The effects of $\mathrm{NO}_{3}^{-}$supplementation on exercise performance in non-athletes appear to be more robust in evaluations using longduration, open-ended tests rather than time trials. Time-trial tests are the most ecologically valid options to assess performance ${ }^{(6,85)}$. Compared with time trials, constant-power (open-ended) tests are more influenced by psychological factors, such as boredom and motivation $^{(86,87)}$. In addition, open-ended tests are more efficient in measuring endurance capacity rather than exercise performance, which is best measured by time-trial protocols ${ }^{(6,88)}$. 


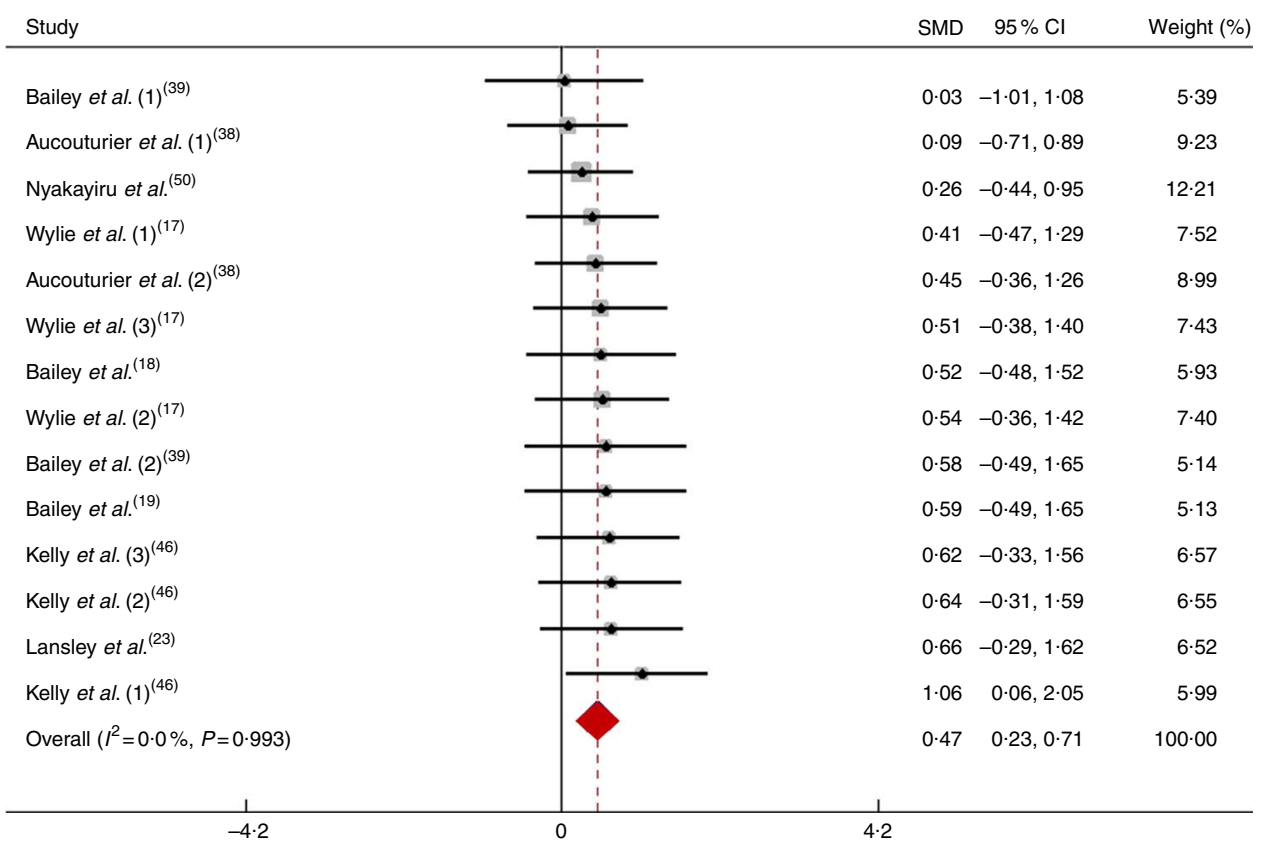

Fig. 9. Forest plot of physical performance during a long-duration open-ended test following dietary $\mathrm{NO}_{3}^{-}$supplementation in non-athletes. SMD, standardised mean difference.

\begin{tabular}{|c|c|c|c|}
\hline Study & SMD & $95 \% \mathrm{Cl}$ & Weight (\%) \\
\hline Murphy et al. ${ }^{(49)}$ & 0.04 & $-0.79,0.88$ & 34.5 \\
\hline Porcelli et al. $(3)^{(51)}$ & 0.06 & $-1 \cdot 06,1 \cdot 19$ & $18 \cdot 8$ \\
\hline Porcelli et al. (2) ${ }^{(51)}$ & $0 \cdot 11$ & $-0.94,1 \cdot 16$ & $21 \cdot 9$ \\
\hline Porcelli et al. $(1)^{(51)}$ & 0.29 & $-0 \cdot 70,1 \cdot 27$ & $24 \cdot 7$ \\
\hline Overall $\left(I^{2}=0.0 \%, P=0.984\right)$ & 0.12 & $-0.37,0.61$ & $100 \cdot 00$ \\
\hline
\end{tabular}

Fig. 10. Forest plot of physical performance during a long-duration time trial following dietary $\mathrm{NO}_{3}^{-}$supplementation in non-athletes. SMD, standardised mean difference.

\begin{tabular}{|c|c|c|c|}
\hline Study & SMD & $95 \% \mathrm{Cl}$ & Weight (\%) \\
\hline Vanhatalo et al. ${ }^{(16)}$ & -0.12 & $-1 \cdot 10,0 \cdot 86$ & $15 \cdot 41$ \\
\hline Vasconcellos et al. ${ }^{(55)}$ & -0.01 & $-0 \cdot 79,0.78$ & 24.09 \\
\hline Wylie et al. ${ }^{(56)}$ & 0.23 & $-0.51,0.97$ & 26.84 \\
\hline Larsen et al. ${ }^{(15)}$ & 0.43 & $-0.51,1 \cdot 36$ & 16.94 \\
\hline Breese et al. ${ }^{(40)}$ & 0.53 & $-0 \cdot 41,1 \cdot 48$ & $16 \cdot 71$ \\
\hline Overall $\left(I^{2}=0.0 \%, P=0.847\right)$ & 0.20 & $-0.18,0.59$ & $100 \cdot 00$ \\
\hline
\end{tabular}

Fig. 11. Forest plot of physical performance during a long-duration graded-exercise test following dietary $\mathrm{NO}_{3}^{-}$supplementation in non-athletes. SMD, standardised mean difference. 


\begin{tabular}{|c|c|c|c|}
\hline Study & SMD & $95 \% \mathrm{Cl}$ & Weight (\%) \\
\hline Lansley et al. (3) & $-0 \cdot 40$ & $-1 \cdot 34,0.52$ & $2 \cdot 05$ \\
\hline Lane et al. $(1)^{(64)}$ & -0.19 & $-0.99,0.61$ & $2 \cdot 78$ \\
\hline McQuillan et al. (2) ${ }^{(67)}$ & -0.14 & $-1 \cdot 06,0 \cdot 78$ & 2.09 \\
\hline Lane et al. (2) & -0.13 & $-0.93,0.66$ & $2 \cdot 79$ \\
\hline McQuillan et al. (1) ${ }^{(67)}$ & -0.12 & $-1.04,0.80$ & 2.09 \\
\hline Cermak et al. (1) ${ }^{(27)}$ & $-0 \cdot 10$ & $-0.72,0.51$ & $4 \cdot 66$ \\
\hline Cermak et al. (2) $)^{(27)}$ & -0.09 & $-0.71,0.52$ & $4 \cdot 66$ \\
\hline McQuillan et al. $(3)^{(67)}$ & -0.09 & $-1 \cdot 01,0.82$ & 2.09 \\
\hline McQuillan et al. (5) & -0.09 & $-1 \cdot 01,0.83$ & 2.09 \\
\hline McQuillan et al. (6) & -0.07 & $-0.99,0.85$ & $2 \cdot 09$ \\
\hline McQuillan et al. (4) ${ }^{(67)}$ & -0.07 & $-0.99,0.85$ & 2.09 \\
\hline Christensen et al. (4) & -0.05 & $-0.97,0.87$ & $2 \cdot 09$ \\
\hline Lane et al. (3) & 0.00 & $-0.80,0.80$ & $2 \cdot 80$ \\
\hline Lane et al. $(4)^{(64)}$ & 0.00 & $-0.80,0.80$ & $2 \cdot 80$ \\
\hline Christensen et al. (1) & 0.00 & $-0.87,0.87$ & $2 \cdot 33$ \\
\hline McQuillan et al. $(7)^{(67)}$ & 0.00 & $-0.92,0.92$ & $2 \cdot 10$ \\
\hline Glaister et al. ${ }^{(60)}$ & 0.02 & $-0.71,0.76$ & $3 \cdot 26$ \\
\hline McQuillan et al. (8) & 0.02 & $-0.89,0.95$ & $2 \cdot 09$ \\
\hline Bescós et al. (2) & 0.03 & $-0.73,0.80$ & 3.03 \\
\hline Callahan et al. (1) & 0.03 & $-0.94,1.01$ & 1.86 \\
\hline Callahan et al. (2) & 0.03 & $-0.94,1.01$ & 1.86 \\
\hline McQuillan et al. (1) & 0.08 & $-0.89,1.06$ & $1 \cdot 86$ \\
\hline Bescós et al. (1) & 0.08 & $-0.68,0.85$ & 3.03 \\
\hline Hoon et al. (2) $)^{(61)}$ & 0.11 & $-0.40,0.63$ & 6.52 \\
\hline Christensen et al. (3) ${ }^{(52)}$ & $0 \cdot 11$ & $-0.76,0.99$ & $2 \cdot 32$ \\
\hline Wilkerson et al. (2) ${ }^{(76)}$ & 0.12 & $-0 \cdot 85,1 \cdot 10$ & $1 \cdot 86$ \\
\hline McQuillan et al. $(2)^{(68)}$ & $0 \cdot 12$ & $-0.85,1 \cdot 10)$ & 1.86 \\
\hline Hoon et al. $(1)^{(61)}$ & $0 \cdot 12$ & $-0.39,0.65$ & 6.52 \\
\hline Cermak et al. (2) & 0.14 & $-0.65,0.94$ & $2 \cdot 79$ \\
\hline Cermak et al. (1) & 0.16 & $-0.63,0.96$ & $2 \cdot 78$ \\
\hline Nyakayiru et al. $(1)^{(50)}$ & $0 \cdot 18$ & $-0 \cdot 48,0.86$ & 3.94 \\
\hline Wilkerson et al. $(1)^{(76)}$ & 0.19 & $-0 \cdot 78,1 \cdot 18$ & 1.85 \\
\hline Bescós et al. ${ }^{(26)}$ & 0.23 & $-0.60,1.07$ & $2 \cdot 54$ \\
\hline Christensen et al. (3) ${ }^{(42)}$ & 0.23 & $-0 \cdot 69,1 \cdot 16$ & $2 \cdot 08$ \\
\hline Lansley et al. (2) & 0.27 & $-0.65,1 \cdot 20$ & $2 \cdot 07$ \\
\hline Lansley et al. (4) ${ }^{(22)}$ & 0.32 & $-0.60,1 \cdot 25$ & $2 \cdot 07$ \\
\hline Lansley et al. (1) & 0.46 & $-0 \cdot 47,1 \cdot 40$ & 2.03 \\
\hline Overall $\left(I^{2}=0.0 \%, P=1.000\right)$ & 0.04 & $-0.09,0.17$ & $100 \cdot 00$ \\
\hline$\perp$ & & & \\
\hline
\end{tabular}

Fig. 12. Forest plot of physical performance in cyclists following dietary $\mathrm{NO}_{3}^{-}$supplementation. $\mathrm{SMD}$, standardised mean difference.

Although the dietary $\mathrm{NO}_{3}^{-}$supplementation did not exert positive effects on the performance of athletes as previously described, the use of this supplement in sports competitions may still be applicable. During competitions, the winner is often determined by narrow differences between athletes, thus creating opportunities for the implementation of practices that may have subtle improvements in performance. Therefore, the distinct sensitivity of different athletes to supplementation should not be disregarded ${ }^{(7,76)}$ and further research on this topic is warranted.

It is important to understand the physiological meaning of the doses that were supplemented in the included studies. These doses ranged from 4.0 to $19.5 \mathrm{mmol}$ (Tables 1 and 2). Considering that the daily ingestion of $\mathrm{NO}_{3}^{-}$corresponds on average to $91 \mathrm{mg}(1.5 \mathrm{mmol})$ in people from the $\mathrm{UK}^{(89)}$, the supplementation would increase the daily ingestion of nitrate by 3 - to 13-fold in this population. However, the dose of the $\mathrm{NO}_{3}^{-}$supplementation, the number of days of supplementation and the total amount ingested do not appear to influence the effects of $\mathrm{NO}_{3}^{-}$supplementation on physical performance in non-athletes and athletes as shown by the lack of significant associations between these parameters. Studies using a single dose showed that $\mathrm{NO}_{3}^{-}$supplementation had either no effects ${ }^{(41,43,45,47)}$ or positive effects ${ }^{(17,53,56)}$ on exercise performance. Likewise,

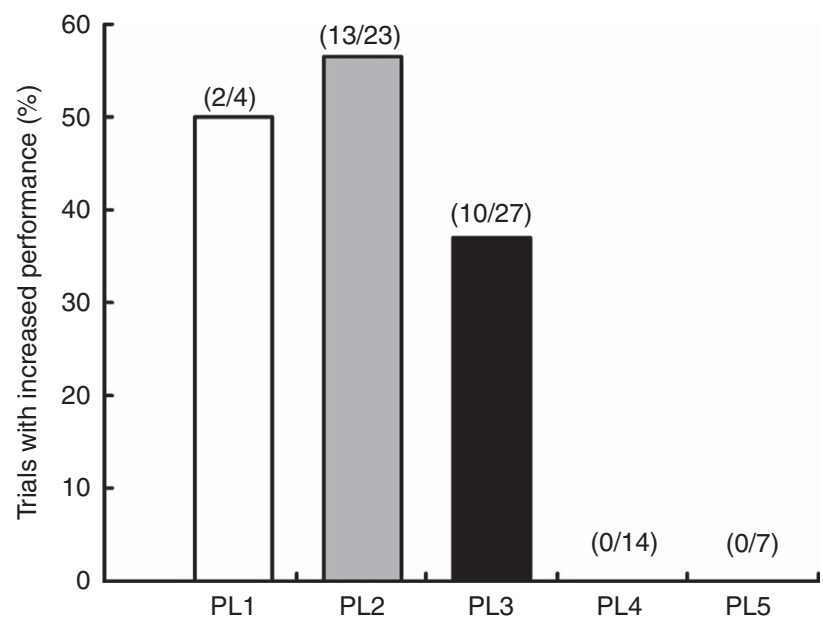

Fig. 13. Number of trials with increased performance (\%) in subjects with different performance levels (PL).

studies using several days ( $\geq 5 \mathrm{~d}$ ) of supplementation showed that $\mathrm{NO}_{3}^{-}$supplementation had either no effects ${ }^{(19,39,45,57)}$ or positive effects ${ }^{(18,22,40,46)}$ on exercise performance. A similar rationale can be applied to the supplementation dose, which does not appear to influence physical performance. 


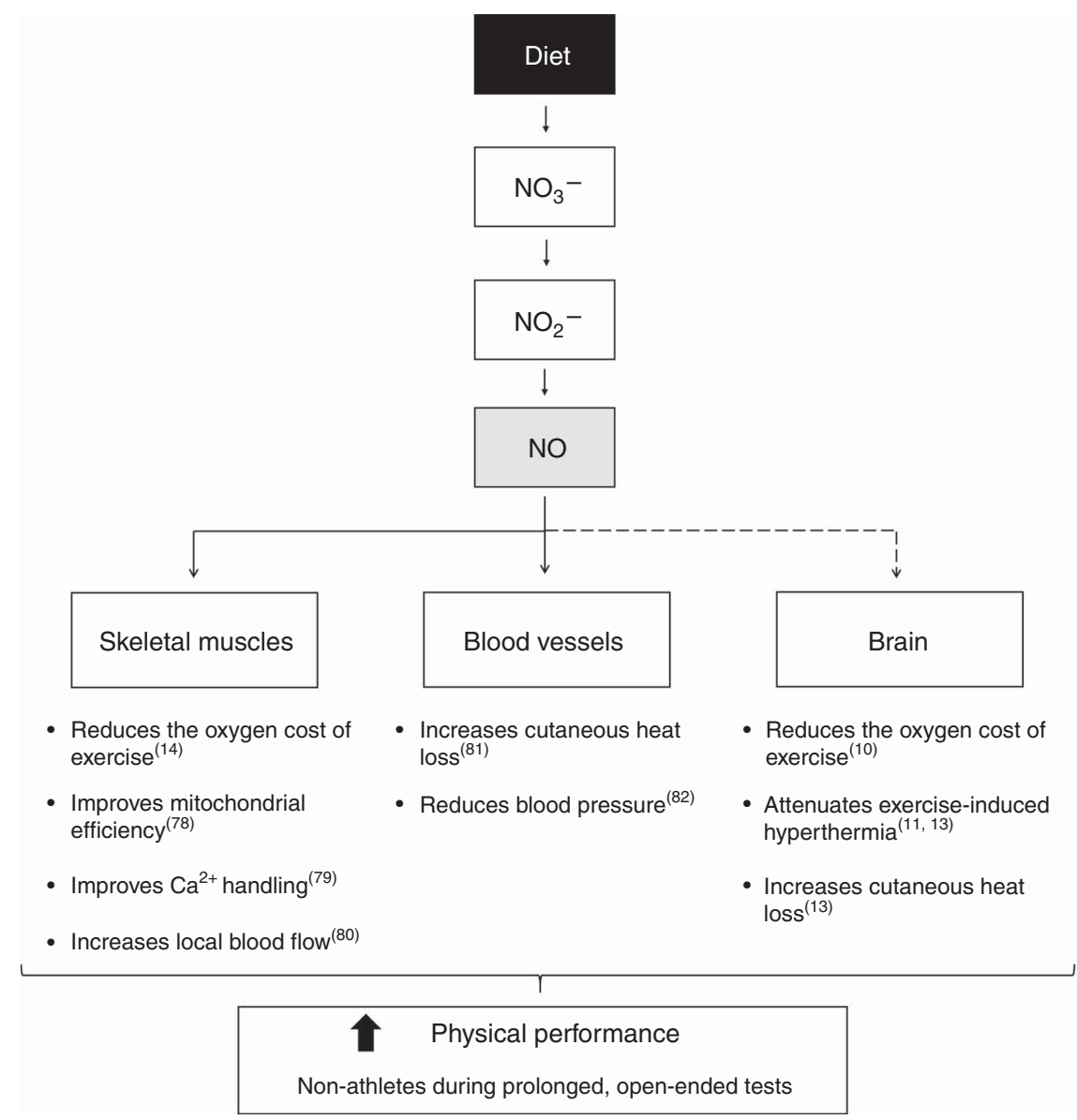

Fig. 14. Mechanisms underlying improved physical performance induced by nitrate $\left(\mathrm{NO}_{3}^{-}\right)$supplementation in non-athletes subjected to prolonged, open-ended tests. Through a series of reduction reactions along the gastrointestinal tract and at target tissues, $\mathrm{NO}_{3}^{-}$acts as the main nitric oxide (NO) donor. Increased $\mathrm{NO}$ bioavailability promotes beneficial effects on performance through effects in skeletal muscles, blood vessels and likely in the brain. To date, no study has provided direct evidence showing that $\mathrm{NO}_{3}^{-}$supplementation increases brain $\mathrm{NO}$ levels (this is the reason why a dashed line is connecting NO to the brain in the schematic). In the skeletal muscles, NO reduces the oxygen cost of exercise ${ }^{(14)}$, improves mitochondrial efficiency ${ }^{(78)}$ and $\mathrm{Ca}^{2+}$ handling ${ }^{(79)}$ and increases local blood flow ${ }^{(80)}$. In the blood vessels, $\mathrm{NO}$ increases cutaneous heat loss ${ }^{(81)}$ and reduces blood pressure ${ }^{(82)}$. Experiments conducted in rats showed that NO in the brain reduces the oxygen cost of exercise ${ }^{(10)}$, attenuates exercise-induced hyperthermia ${ }^{(11,13)}$ and increases cutaneous heat $\operatorname{loss}^{(13)}$. Collectively, these physiological responses induced by $\mathrm{NO}_{3}^{-}$supplementation improve performance in the conditions mentioned above.

For example, studies using low doses $(4-5.5 \mathrm{mmol})$ showed that $\mathrm{NO}_{3}^{-}$supplementation had either no effects ${ }^{(17,19,38,51)}$ or positive effects $^{(16,51,53,56)}$ on exercise performance. Finally, studies using high doses $(>10 \mathrm{mmol})$ also showed that $\mathrm{NO}_{3}^{-}$supplementation had no effects ${ }^{(43,45,52)}$ or positive effects ${ }^{(17,43)}$ on exercise performance.

Despite the high variability in the experimental protocols used in the studies analysed in the present review, the analysed subgroups did not include heterogeneous samples. Therefore, the data homogeneity, the quality of the studies assessed by the risk of bias and the absence of publication bias in the studies used in this systematic review and meta-analysis are sufficient to draw conclusions.

A major limitation of this review is related to the wide variation in the methods (differences in the dose of $\mathrm{NO}_{3}^{-}$, number of days of supplementation, total amount ingested and mode of $\mathrm{NO}_{3}^{-}$ delivery) used in the analysed studies. This methodological diversity complicates the interpretation of the results and precludes clear conclusions regarding certain features of supplementation, such as those listed above. In addition, most studied individuals were men, and whether a sex-related sensitivity to the enhancing effects of nitrate exists in nonathletes is unclear. Thus, future studies should include women as participants.

\section{Practical applications}

The present results may encourage coaches, athletes and exercise practitioners to consider the following: (1) $\mathrm{NO}_{3}^{-}$ supplementation appears to be more effective in non-athletes than in athletes, particularly in performance evaluations using long-duration, open-ended tests; (2) the ergogenic effects mediated by $\mathrm{NO}_{3}^{-}$supplementation do not affect physical performance in athletes, including cyclists, which are the most studied athletic population; and (3) subjects classified at a lower PL (i.e. less conditioned) are more responsive to the effects of $\mathrm{NO}_{3}^{-}$supplementation than are subjects classified at a higher PL. 


\section{Conclusion}

The present systematic review and meta-analysis indicates that dietary $\mathrm{NO}_{3}^{-}$supplementation improves physical performance in non-athletes, particularly in performance evaluations using long-duration, open-ended tests. In contrast, dietary $\mathrm{NO}_{3}^{-}$ supplementation does not appear to benefit the performance of athletes.

\section{Acknowledgements}

The authors thank Dr Luc Van Loon for his helpful comments to the first draft of this manuscript.

This study was supported by Conselho Nacional de Desenvolvimento Científico e Tecnológico (CNPq), Coordenação de Aperfeiçoamento de Pessoal de Nível Superior (CAPES) and Fundação de Amparo à Pesquisa do Estado de Minas Gerais (FAPEMIG). The funding institutions had no role in the study design, data analysis, decision to publish or preparation of the article.

All authors contributed to the development of the research question and study design. H. O. C., L. R. D., Q. T. R. and W. P. performed the literature search. H. O. C., L. R. D., Q. T. R. and F. S. M. M. performed the study selection. H. O. C., L. R. D. and Q. T. R. analysed the data. All authors interpreted the results and wrote the manuscript. All authors reviewed and approved the manuscript.

The authors declare that there are no conflicts of interest.

\section{Supplementary material}

For supplementary material/s referred to in this article, please visit http://dx.doi.org/10.1017/S0007114518000132

\section{References}

1. Jones AM, Haramizu S, Ranchordas M, et al. (2011) A-Z of nutritional supplements: dietary supplements, sports nutrition foods and ergogenic aids for health and performance part 27. BrJ Sports Med 45, 1246-1248.

2. Jones AM, Vanhatalo A \& Bailey SJ (2013) Influence of dietary nitrate supplementation on exercise tolerance and performance. Nestle Nutr Inst Workshop Ser 75, 27-40.

3. Jones AM (2014) Dietary nitrate supplementation and exercise performance. Sports Med 44, Suppl. 1, S35-S45.

4. Thompson C, Wylie LJ, Blackwell JR, et al. (2017) Influence of dietary nitrate supplementation on physiological and muscle metabolic adaptations to sprint interval training. I Appl Physiol (1985) 122, 642-652.

5. Hoon MW, Johnson NA, Chapman PG, et al. (2013) The effect of nitrate supplementation on exercise performance in healthy individuals: a systematic review and meta-analysis. Int J Sport Nutr Exerc Metab 23, 522-532.

6. McMahon NF, Leveritt MD \& Pavey TG (2017) The effect of dietary nitrate supplementation on endurance exercise performance in healthy adults: a systematic review and metaanalysis. Sports Med 47, 735-756.

7. Jonvik KL, Nyakayiru J, van Loon LJ, et al. (2015) Last word on viewpoint: can elite athletes benefit from dietary nitrate supplementation? J Appl Physiol (1985) 119, 770.
8. Jonvik KL, Nyakayiru J, van Loon LJ, et al. (2015) Can elite athletes benefit from dietary nitrate supplementation? I Appl Physiol (1985) 119, 759-761.

9. Hultstrom M, Amorim de Paula C, Antonio Peliky Fontes M, et al. (2015) Commentaries on viewpoint: can elite athletes benefit from dietary nitrate supplementation? J Appl Physiol (1985) 119, 762-769.

10. Lacerda AC, Marubayashi U, Balthazar CH, et al. (2006) Evidence that brain nitric oxide inhibition increases metabolic cost of exercise, reducing running performance in rats. Neurosci Lett 393, 260-263.

11. Lacerda AC, Marubayashi U \& Coimbra CC (2005) Nitric oxide pathway is an important modulator of heat loss in rats during exercise. Brain Res Bull 67, 110-116.

12. Lima PM, Santiago HP, Szawka RE, et al. (2014) Central blockade of nitric oxide transmission impairs exercise-induced neuronal activation in the PVN and reduces physical performance. Brain Res Bull 108, 80-87.

13. Wanner SP, Leite LH, Guimaraes JB, et al. (2015) Increased brain L-arginine availability facilitates cutaneous heat loss induced by running exercise. Clin Exp Pharmacol Physiol 42, 609-616.

14. Larsen FJ, Weitzberg E, Lundberg JO, et al. (2007) Effects of dietary nitrate on oxygen cost during exercise. Acta Physiol 191, 59-66.

15. Larsen FJ, Weitzberg E, Lundberg JO, et al. (2010) Dietary nitrate reduces maximal oxygen consumption while maintaining work performance in maximal exercise. Free Radic Biol Med 48, 342-347.

16. Vanhatalo A, Bailey SJ, Blackwell JR, et al. (2010) Acute and chronic effects of dietary nitrate supplementation on blood pressure and the physiological responses to moderateintensity and incremental exercise. Am J Physiol Regul Integr Comp Physiol 299, R1121-R1131.

17. Wylie LJ, Kelly J, Bailey SJ, et al. (2013) Beetroot juice and exercise: pharmacodynamic and dose-response relationships. J Appl Physiol (1985) 115, 325-336.

18. Bailey SJ, Winyard P, Vanhatalo A, et al. (2009) Dietary nitrate supplementation reduces the $\mathrm{O}_{2}$ cost of low-intensity exercise and enhances tolerance to high-intensity exercise in humans. J Appl Physiol 107, 1144-1155.

19. Bailey SJ, Fulford J, Vanhatalo A, et al. (2010) Dietary nitrate supplementation enhances muscle contractile efficiency during knee-extensor exercise in humans. J Appl Physiol 109, 135-148.

20. Bond H, Morton L \& Braakhuis AJ (2012) Dietary nitrate supplementation improves rowing performance in welltrained rowers. Int J Sport Nutr Exerc Metab 22, 251-256.

21. Cermak NM, Gibala MJ \& van Loon LJC (2012) Nitrate supplementation's improvement of 10-km time-trial performance in trained cyclists. Int J Sport Nutr Exerc Metab 22, 64-71.

22. Lansley KE, Winyard PG, Bailey SJ, et al. (2011) Acute dietary nitrate supplementation improves cycling time trial performance. Med Sci Sports Exerc 43, 1125-1131.

23. Lansley KE, Winyard PG, Fulford J, et al. (2011) Dietary nitrate supplementation reduces the $\mathrm{O}_{2}$ cost of walking and running: a placebo-controlled study. J Appl Physiol 110, 591-600.

24. Masschelein E, Van Thienen R, Wang X, et al. (2012) Dietary nitrate improves muscle but not cerebral oxygenation status during exercise in hypoxia. J Appl Physiol (1985) 113, 736-745.

25. Bescós R, Ferrer-Roca V, Galilea PA, et al. (2012) Sodium nitrate supplementation does not enhance performance of endurance athletes. Med Sci Sports Exerc 44, 2400-2409.

26. Bescós R, Rodriguez FA, Iglesias X, et al. (2011) Acute administration of inorganic nitrate reduces VO(2peak) in endurance athletes. Med Sci Sports Exerc 43, 1979-1986. 
27. Cermak NM, Res P, Stinkens R, et al. (2012) No improvement in endurance performance after a single dose of beetroot juice. Int J Sport Nutr Exerc Metab 22, 470-478.

28. Peacock O, Tjonna AE, James P, et al. (2012) Dietary nitrate does not enhance running performance in elite crosscountry skiers. Med Sci Sports Exerc 44, 2213-2219.

29. Wilkerson DP, Hayward G, Stephen BJ, et al. (2012) Acute dietary nitrate supplementation does not improve 50-mile time trial performance in highly trained cyclists. Med Sci Sport Exerc 44, 442-442.

30. Liberati A, Altman DG, Tetzlaff J, et al. (2009) The PRISMA statement for reporting systematic reviews and meta-analyses of studies that evaluate health care interventions: explanation and elaboration. Ann Intern Med 151, W65-W94.

31. Moher D, Liberati A, Tetzlaff J, et al. (2009) Preferred reporting items for systematic reviews and meta-analyses: the PRISMA statement. Ann Intern Med 151, 264-269, W264.

32. Christensen PM, Nyberg M \& Bangsbo J (2013) Influence of nitrate supplementation on $\mathrm{VO}_{2}$ kinetics and endurance of elite cyclists. Scand J Med Sci Sports 23, e21-e31.

33. Duffield R, Dawson B \& Goodman C (2005) Energy system contribution to 400-metre and 800-metre track running. J Sports Sci 23, 299-307.

34. De Pauw K, Roelands B, Cheung SS, et al. (2013) Guidelines to classify subject groups in sport-science research. Int J Sports Physiol Perform 8, 111-122.

35. JPT Higgins \& Green S (editors) (2011) Cochrane Handbook for Systematic Reviews of Interventions, version 5.1.0 (updated March 2011). The Cochrane Collaboration. http://handbook. cochrane.org

36. Cohen J (1988) Statistical Power Analysis for the Behavioral Sciences, 2nd ed. Hillsdale, NJ: Lawrence Earlbaum Associates.

37. Sterne JA, Sutton AJ, Ioannidis JP, et al. (2011) Recommendations for examining and interpreting funnel plot asymmetry in meta-analyses of randomised controlled trials. BMJ 343, d 4002 .

38. Aucouturier J, Boissiere J, Pawlak-Chaouch M, et al. (2015) Effect of dietary nitrate supplementation on tolerance to supramaximal intensity intermittent exercise. Nitric Oxide 49, 16-25.

39. Bailey SJ, Varnham RL, DiMenna FJ, et al. (2015) Inorganic nitrate supplementation improves muscle oxygenation, $\mathrm{O}_{2}$ uptake kinetics, and exercise tolerance at high but not low pedal rates. J Appl Physiol 118, 1396-1405.

40. Breese BC, McNarry MA, Marwood S, et al. (2013) Beetroot juice supplementation speeds $\mathrm{O}_{2}$ uptake kinetics and improves exercise tolerance during severe-intensity exercise initiated from an elevated metabolic rate. Am J Physiol Regul Integr Comp Physiol 305, R1441-R1450.

41. Buck CL, Henry T, Guelfi K, et al. (2015) Effects of sodium phosphate and beetroot juice supplementation on repeatedsprint ability in females. Eur J Appl Physiol 115, 2205-2213.

42. Christensen PM, Petersen NK, Friis SN, et al. (2017) Effects of nitrate supplementation in trained and untrained muscle are modest with initial high plasma nitrite levels. Scand J Med Sci Sports 27, 1616-1626.

43. Coggan AR, Leibowitz JL, Kadkhodayan A, et al. (2015) Effect of acute dietary nitrate intake on maximal knee extensor speed and power in healthy men and women. Nitric Oxide 48, 16-21.

44. Corry LR \& Gee TI (2015) Dietary nitrate enhances power output during the early phases of maximal intensity sprint cycling. Int J Coaching Sci 9, 87-97.

45. Fulford J, Winyard PG, Vanhatalo A, et al. (2013) Influence of dietary nitrate supplementation on human skeletal muscle metabolism and force production during maximum voluntary contractions. Pflugers Arch 465, 517-528.
46. Kelly J, Vanhatalo A, Wilkerson DP, et al. (2013) Effects of nitrate on the power-duration relationship for severe-intensity exercise. Med Sci Sports Exerc 45, 1798-1806.

47. Kokkinoplitis K \& Chester N (2014) The effect of beetroot juice on repeated sprint performance and muscle force production. J Phys Educ Sport 14, 242-247.

48. Mosher SL, Sparks SA, Williams EL, et al. (2016) Ingestion of a nitric oxide enhancing supplement improves resistance exercise performance. J Strength Cond Res 30, 3520-3524.

49. Murphy M, Eliot K, Heuertz RM, et al. (2012) Whole beetroot consumption acutely improves running performance. $J$ Acad Nutr Diet 112, 548-552.

50. Nyakayiru J, Jonvik KL, Trommelen J, et al. (2017) Beetroot juice supplementation improves high-intensity intermittent type exercise performance in trained soccer players. Nutrients $\mathbf{9}, \mathrm{E} 314$.

51. Porcelli S, Ramaglia M, Bellistri G, et al. (2015) Aerobic fitness affects the exercise performance responses to nitrate supplementation. Med Sci Sports Exerc 47, 1643-1651.

52. Rienks JN, Vanderwoude AA, Maas E, et al. (2015) Effect of beetroot juice on moderate-intensity exercise at a constant rating of perceived exertion. Int J Exerc Sci 8, 277-286.

53. Thompson KG, Turner L, Prichard J, et al. (2014) Influence of dietary nitrate supplementation on physiological and cognitive responses to incremental cycle exercise. Respir Physiol Neurobiol 193, 11-20.

54. Thompson C, Wylie LJ, Fulford J, et al. (2015) Dietary nitrate improves sprint performance and cognitive function during prolonged intermittent exercise. Eur J Appl Physiol 115, 1825-1834.

55. Vasconcellos J, Henrique Silvestre D, Dos Santos Baiao D, et al. (2017) A single dose of beetroot gel rich in nitrate does not improve performance but lowers blood glucose in physically active individuals. J Nutr Metabol 2017, 7853034.

56. Wylie LJ, Mohr M, Krustrup P, et al. (2013) Dietary nitrate supplementation improves team sport-specific intense intermittent exercise performance. Eur J Appl Physiol 113, 1673-1684.

57. Wylie LJ, Bailey SJ, Kelly J, et al. (2016) Influence of beetroot juice supplementation on intermittent exercise performance. Eur J Appl Physiol 116, 415-425.

58. Boorsma RK, Whitfield J \& Spriet LL (2014) Beetroot juice supplementation does not improve performance of elite 1500-m runners. Med Sci Sports Exerc 46, 2326-2334.

59. Callahan MJ, Parr EB, Hawley JA, et al. (2017) Single and combined effects of beetroot crystals and sodium bicarbonate on 4-km cycling time trial performance. Int J Sport Nutr Exerc Metab 27, 271-278.

60. Glaister M, Pattison JR, Muniz-Pumares D, et al. (2015) Effects of dietary nitrate, caffeine, and their combination on $20-\mathrm{km}$ cycling time trial performance. J Strength Cond Res 29, 165-174.

61. Hoon MW, Hopkins WG, Jones AM, et al. (2014) Nitrate supplementation and high-intensity performance in competitive cyclists. Appl Physiol Nutr Metab 39, 1043-1049.

62. Hoon MW, Jones AM, Johnson NA, et al. (2014) The effect of variable doses of inorganic nitrate-rich beetroot juice on simulated 2000-m rowing performance in trained athletes. Int J Sport Physiol 9, 615-620.

63. Kramer SJ, Baur DA, Spicer MT, et al. (2016) The effect of six days of dietary nitrate supplementation on performance in trained CrossFit athletes. J Int Soc Sports Nutr 13, 39.

64. Lane SC, Hawley JA, Desbrow B, et al. (2014) Single and combined effects of beetroot juice and caffeine supplementation on cycling time trial performance. Appl Physiol Nutr Metab 39, 1050-1057. 
65. Lowings S, Shannon OM, Deighton K, et al. (2017) Effect of dietary nitrate supplementation on swimming performance in trained swimmers. Int J Sport Nutr Exerc Metab 27, 377-384.

66. Martin K, Smee D, Thompson KG, et al. (2014) No improvement of repeated-sprint performance with dietary nitrate. Int J Sports Physiol Perform 9, 845-850.

67. McQuillan JA, Dulson DK, Laursen PB, et al. (2017) Dietary nitrate fails to improve 1 and $4 \mathrm{~km}$ cycling performance in highly trained cyclists. Int J Sport Nutr Exerc Metab 27, 255-263.

68. McQuillan JA, Dulson DK, Laursen PB, et al. (2017) The effect of dietary nitrate supplementation on physiology and performance in trained cyclists. Int J Sports Physiol Perform 12, 684-689.

69. Muggeridge DJ, Howe CC, Spendiff O, et al. (2013) The effects of a single dose of concentrated beetroot juice on performance in trained flatwater kayakers. Int J Sport Nutr Exerc Metab 23, 498-506.

70. Nyakayiru JM, Jonvik KL, Pinckaers PJ, et al. (2017) No effect of acute and 6-day nitrate supplementation on $\mathrm{VO}_{2}$ and timetrial performance in highly trained cyclists. Int J Sport Nutr Exerc Metab 27, 11-17.

71. Peeling P, Cox GR, Bullock N, et al. (2015) Beetroot juice improves on-water $500 \mathrm{M}$ time-trial performance, and laboratorybased paddling economy in national and international-level Kayak athletes. Int J Sport Nutr Exerc Metab 25, 278-284.

72. Rimer EG, Peterson LR, Coggan AR, et al. (2016) Acute dietary nitrate supplementation increases maximal cycling power in athletes. Int J Sports Physiol Perform 11, 715-720.

73. Rimer EG, Peterson LR, Coggan AR, et al. (2016) Increase in maximal cycling power with acute dietary nitrate supplementation. Int J Sports Physiol Perform 11, 715-720.

74. Shannon OM, Barlow MJ, Duckworth L, et al. (2017) Dietary nitrate supplementation enhances short but not longer duration running time-trial performance. Eur J Appl Physiol 117, 775-785.

75. Thompson C, Vanhatalo A, Jell H, et al. (2016) Dietary nitrate supplementation improves sprint and high-intensity intermittent running performance. Nitric Oxide 61, 55-61.

76. Wilkerson DP, Hayward GM, Bailey SJ, et al. (2012) Influence of acute dietary nitrate supplementation on 50 mile time trial performance in well-trained cyclists. Eur J Appl Physiol 112, $4127-4134$.
77. Goulet ED (2011) Effect of exercise-induced dehydration on time-trial exercise performance: a meta-analysis. $\mathrm{Br} J$ Sports Med 45, 1149-1156.

78. Larsen FJ, Schiffer TA, Borniquel S, et al. (2011) Dietary inorganic nitrate improves mitochondrial efficiency in humans. Cell Metab 13, 149-159.

79. Hernández A, Schiffer TA, Ivarsson N, et al. (2012) Dietary nitrate increases tetanic $\left[\mathrm{Ca}^{2+}\right] \mathrm{i}$ and contractile force in mouse fast-twitch muscle. J Physiol 590, 3575-3583.

80. Ferguson SK, Hirai DM, Copp SW, et al. (2013) Impact of dietary nitrate supplementation via beetroot juice on exercising muscle vascular control in rats. J Physiol 591, 547-557.

81. McNamara TC, Keen JT, Simmons GH, et al. (2014) Endothelial nitric oxide synthase mediates the nitric oxide component of reflex cutaneous vasodilatation during dynamic exercise in humans. J Physiol 592, 5317-5326.

82. Larsen FJ, Ekblom B, Sahlin K, et al. (2006) Effects of dietary nitrate on blood pressure in healthy volunteers. New Eng J Med 355, 2792-2793.

83. McConell GK, Bradley SJ, Stephens TJ, et al. (2007) Skeletal muscle nNOS mu protein content is increased by exercise training in humans. Am J Physiol Regul Integr Comp Physiol 293, R821-R828.

84. Tesch PA \& Karlsson J (1985) Muscle fiber types and size in trained and untrained muscles of elite athletes. J Appl Physiol (1985) 59, 1716-1720.

85. Currell K \& Jeukendrup AE (2008) Validity, reliability and sensitivity of measures of sporting performance. Sports Med 38, 297-316.

86. Amann M, Hopkins WG \& Marcora SM (2008) Similar sensitivity of time to exhaustion and time-trial time to changes in endurance. Med Sci Sports Exerc 40, 574-578.

87. Jeukendrup A, Saris WH, Brouns F, et al. (1996) A new validated endurance performance test. Med Sci Sports Exerc 28, 266-270.

88. Jeukendrup AE \& Currell K (2005) Should time trial performance be predicted from three serial time-to-exhaustion tests? Med Sci Sports Exerc 37, 1820 author reply 1821.

89. Alexander J, Diane B, Cockburn A, et al. (2008) Nitrate in vegetables scientific opinion of the Panel on Contaminants in the Food chain. EFSA J 689, 1-79. 